\title{
1. QUANTITATIVE NANNOFOSSIL BIOSTRATIGRAPHY OF QUATERNARY SEQUENCES FROM GUYOTS IN THE CENTRAL AND WESTERN PACIFIC OCEAN ${ }^{1}$
}

\author{
Elisabetta Erba ${ }^{2}$
}

\begin{abstract}
Quantitative analysis of calcareous nannofossils was performed on Quaternary sediments recovered during Leg 144 at Sites 880 (Takuyo-Daisan Guyot), 871 (Limalok Guyot), 872 (Lo-En Guyot), and 873 (Wodejebato Guyot). For each sample, at least 500 specimens were counted with the light microscope and the relative abundance of taxa was computed as percentages. Calcareous nannofloras are very abundant and well preserved. Assemblages are dominated by the gephyrocapsid group, which was subdivided using a simplified taxonomy based on morphologic and morphometric characters that are easily applicable during light microscope investigations. Quantitative analysis of nannofloras revealed distinctive changes in the abundance patterns, especially within the Gephyrocapsa group, at all sites. These events seem to be coeval on a global scale and were used for high-resolution biostratigraphy. Most nannofossil events and biozones previously proposed for the Quaternary were recognized.

The sequence recovered at Site 880 is almost complete, but a hiatus was detected in the upper Pleistocene, where the $G$. oceanica Zone is extremely short. At Site 871 , the Quaternary sequence seems to be complete and all the nannofossil events and biozones were detected. Also at Site 872, a complete sequence of nannofossil events was recognized, but the Quaternary section is very thin and suggests very low sedimentation rates and/or the occurrence of minor hiatuses that cannot be determined with biostratigraphy. The spacing of nannofossil events at Sites 800,871 , and 872 is quite different, suggesting variable sedimentation rates and minor gaps caused by the strong currents on top of these guyots. At Site 873, most of the Quaternary is missing; detection of only the lower Pleistocene nannofossil events suggests that the accumulation of recent sediments on Wodejebato Guyot is strongly affected by current activity.

Major changes in the composition of Pleistocene nannofloras appear to be coeval on a global scale across a wide latitudinal range within the tropical to equatorial belts. However, the nannofossil records at Sites $871,872,873$, and 880 indicate that some taxa were locally and temporarily influenced by the specific oceanographic conditions. Further investigations on the distribution of specific taxa, such as the smallest specimens of Gephyrocapsa $(2-2.5 \mu \mathrm{m})$ and Florisphaera profunda, may help in separating global vs. local/regional effects.
\end{abstract}

\section{INTRODUCTION}

Ocean Drilling Program (ODP) Leg 144 was devoted to the exploration of guyots in the central and western Pacific, in order to reconstruct the geological history of Cretaceous and Paleogene atolls that progressively subsided to present water depths between 1084 and $1525 \mathrm{~m}$ below seafloor ( $\mathrm{mbsf}$ ) (Table 1 ). The five guyots investigated are aligned along a southeast-northwest transect in a wide latitude band ranging from $5^{\circ} 33.13^{\prime} \mathrm{N}$ to $34^{\circ} 12.53^{\prime} \mathrm{N}$ (see Frontispiece and Table 1). Major objectives of Leg 144 included the construction of the volcanic edifice, the onset of marine sedimentation, and the development of carbonate platforms on the volcanic pedestal; the demise of shallow-water sedimentation; and the Cenozoic history of pelagic sediments capping the guyots.

Tertiary and Quaternary pelagic caps were recovered on Limalok Guyot (Site 871), Lo-En Guyot (Site 872), Wodejebato Guyot (Site 873), MIT Guyot (Site 878), and Takuyo-Daisan Guyot (Site 880). Shipboard investigations (Premoli Silva, Haggerty, Rack, et al., 1993) revealed that these pelagic sequences are characterized by several unconformities that resulted from the strong currents winnowing the nannofossil-foraminifer oozes on top of the guyots. As the Quaternary appeared to be well represented at most sites, a special study was dedicated to this interval.

This paper documents the calcareous nannofossil distribution in the Quaternary sequences recovered at Sites 871, 872, 873, and 880 . Site 878 was not studied because only a very thin $(3.2 \mathrm{~m})$ Pleistocene to Miocene pelagic section was penetrated. The upper part of this section is extremely soupy and contains mixed nannofossil assem-

\footnotetext{
${ }^{1}$ Haggerty, J.A., Premoli Silva, I., Rack, F., and McNutt, M.K. (Eds.), 1995. Proc $O D P_{2}$ Sci. Results, 144: College Station, TX (Ocean Drilling Program).

Dipartimento di Scienze della Terra, Universita di Milano, Via L. Mangiagalli 34, I-20133 Milano, Italy.
}

blages of late Pliocene and early Pleistocene age that hampered detailed investigations (Premoli Silva, Haggerty, Rack, et al., 1993; Watkins et al., this volume).

A detailed study was applied to Site 880 (Takuyo-Daisan Guyot), which was specifically drilled to recover the pelagic cap on the northernmost guyot of the transect. Although the limited time allocated to this site prevented reaching the contact between the pelagic cap and the underlying platform limestone, a complete Holocene to uppermost Pliocene section was penetrated. The sediments recovered at this site are undisturbed and magnetostratigraphy was obtained (Rack et al., this volume). On the contrary, at Sites 871,872 , and 873 paleomagnetic studies were performed throughout the pelagic caps, but no magnetostratigraphic interpretation was possible owing to the generally soupy texture of the sediments (Premoli Silva, Haggerty, Rack, et al., 1993). Because the Quaternary section at Site 800 yields the most complete data set, with both biostratigraphy and magnetostratigraphy, this site will be presented and discussed first, whereas Sites 871,872 , and 873 will be discussed later.

Because the use of Quaternary nannofossil events allow us to obtain a high-resolution biostratigraphy, the present study will be used to correlate the four investigated sites and estimate possible hiatuses related to current activity vs. accumulation at various guyots in the central and western Pacific. Moreover, changes in nannofloral composition will be used for ecologic/oceanographic interpretations.

\section{MATERIALS AND METHODS}

At Site 880 sampling has a resolution of $20 \mathrm{~cm}$ throughout the core (Table 2), whereas at Sites 871,872 , and 873 samples were analyzed every $50 \mathrm{~cm}$ (Tables $3-5$ ). Raw material was permanently mounted using Norland Optical Adhesive; sediments were not ultrasonically cleaned or centrifuged so that we could retain the original sediment and assemblage composition. A quantitative study of calcareous nannofos- 
Table 1. Latitude, longitude, and water depth of Sites $871,872,873,878$, and 880 drilled during Leg 144.

\begin{tabular}{llccc}
\hline Site & \multicolumn{1}{c}{ Guyot } & $\begin{array}{c}\text { Latitude } \\
(\mathrm{N})\end{array}$ & $\begin{array}{c}\text { Longitude } \\
(\mathrm{E})\end{array}$ & $\begin{array}{c}\text { Water } \\
\text { depth } \\
(\mathrm{m})\end{array}$ \\
\hline 871 & Limalok & $5^{\circ} 33.13^{\prime}$ & $172^{\circ} 20.66^{\prime}$ & 1255 \\
872 & Lo-En & $10^{\circ} 05.85^{\prime}$ & $162^{\circ} 51.96^{\prime}$ & 1084 \\
873 & Wodejebato & $11^{\circ} 53.84^{\prime}$ & $164^{\circ} 55.20^{\prime}$ & 1334 \\
878 & MIT & $27^{\circ} 19.143^{\prime}$ & $151^{\circ} 53.028^{\prime}$ & 1323 \\
880 & Takuyo-Daisan & $34^{\circ} 12.53^{\prime}$ & $144^{\circ} 18.74^{\prime}$ & 1525 \\
\hline
\end{tabular}

sils was performed with the polarizing light microscope, at $1250 \times$ magnification on smear slides prepared using standard techniques. Nannofossils were quantified by counting at least 500 specimens in each sample, and single taxa abundance is reported in Tables 2 through 5 as counts and percentages of total nannoflora. Qualitative investigation was extended to 100 additional fields of view to search for rare but biostratigraphically important taxa.

Because the genus Gephyrocapsa is dominant through the Pleistocene, the composition of this group was quantified as suggested by Raffi et al. (1993). These authors proposed to use relative abundances (percentages) of gephyrocapsid classes in counts of at least 100 gephyrocapsid specimens. In the Quaternary sequences of Sites 880, 871,872 , and 873 , much more than 100 gephyrocapsids were identified in counts of 500 specimens of total nannofloras. Therefore, the percentages of single classes within the genus Gephyrocapsa were calculated on the original counts.

\section{TAXONOMIC REMARKS}

Pleistocene calcareous nannofloras are usually dominated by groups represented by small-sized coccoliths. In particular, the Gephyrocapsa group shows very high abundances and is extensively used for biostratigraphy. However, despite their abundance, there is no standard taxonomy for gephyrocapsids, and very little agreement on their taxonomy and nomenclature exists among biostratigraphers. Discrepancies also result from the use of a scanning electron microscope vs. a light microscope for the analysis of morphologic variations within the genus Gephyrocapsa. Recently, Raffi et al. (1993) discussed the gephyrocapsid taxonomy and the various criteria that have been proposed for the classification of this group (Gartner, 1977; Rio, 1982; Takayama and Sato, 1987; Matsuoka and Okada, 1989). For the present study, the informal, morphometric taxonomic concepts of Rio (1982) and Raffi et al. (1993) were followed. In fact, this simplified taxonomy is readily applicable using the light microscope and was proven to give reliable and reproducible results in the Atlantic and Pacific oceans (Raffi et al., 1993) as well as in the Mediterranean Sea (Rio et al., 1990; Castradori, 1993a).

For the Reticulofenestra group, the morphometric taxonomic concepts proposed by Matsuoka and Okada (1989) were partially adopted, as discussed below.

\section{Gephyrocapsid Group}

In agreement with Rio (1982), for the present study, specimens of the genus Gephyrocapsa were subdivided as follows:

1. Small Gephyrocapsa: specimens with a maximum length shorter than $3.5 \mu \mathrm{m}$;

2. Large Gephyrocapsa: specimens with a maximum length longer than $5.5 \mu \mathrm{m}$;

3. Medium-sized Gephyrocapsa: specimens with a maximum length between 3.5 and $5.5 \mu \mathrm{m}$.

The last subgroup is further subdivided into three species: $G$. caribbeanica, G. oceanica s.1., and Gephyrocapsa sp. 3. G. carib- beanica has a small central area spanned by a short bar. In the material studied, most specimens are 3.5 to $4 \mu \mathrm{m}$ in size. G. oceanica s.l. has an open central area spanned by a diagonal bar (Rio et al., 1990). Gephyrocapsa sp. 3 has an open central area spanned by a bar that is nearly parallel to the short axis of the ellipse $\left(<20^{\circ}\right)$. Gephyrocapsa sp. 3 is a synonym of $G$. omega and $G$. parallela (see Raffi et al., 1993 , for discussion) and is equivalent to "G. oceanica (vertical)" of Matsuoka and Okada (1989).

\section{Reticulofenestrid Group}

The taxonomy of the Pleistocene reticulofenestrids has been discussed by various workers (Pujos, 1985a, 1985b; Takayama and Sato, 1987; Matsuoka and Okada, 1989). For the present study, specimens of the genus Reticulofenestra were subdivided as follows:

1. Small Reticulofenestra: specimens with a maximum length shorter than $4 \mu \mathrm{m}$;

2. Medium-sized Reticulofenestra: specimens with a maximum length between 4 and $6.5 \mu \mathrm{m}$;

3. Large Reticulofenestra: specimens with a maximum length between 6.5 and $8 \mu \mathrm{m}$.

The latter specimens are close to $R$. asanoi of Sato and Takayama (1990), but most specimens are elliptical and not circular.

In all of the tables the small- and the medium-sized Reticulofenestra are reported in the same column because they are equally abundant in the lower Pleistocene and are mainly represented by specimens close to $4 \mu \mathrm{m}$ in size. The large Reticulofenestra group is comparable to the "Reticulofenestra A (large variety)" of Matsuoka and Okada (1989).

\section{Pseudoemiliania lacunosa Group}

Pseudoemiliania lacunosa is abundant in the lower Pleistocene and fluctuates in abundance before its disappearance in the middle Pleistocene. In the present study, specimens were subdivided as follows:

1. P. lacunosa E: elliptical specimens;

2. P. lacunosa $\mathrm{C}$ : circular specimens.

In all the Quaternary sequences at Sites $880,871,872$, and 873 , specimens of the $P$. lacunosa $\mathrm{E}$ group are much more abundant than specimens of the $P$. lacunosa $\mathrm{C}$ group, but their stratigraphic range is identical (Tables 2-5).

\section{RESULTS}

Very abundant nannofossil assemblages were observed throughout the Quaternary sequences at Sites $880,871,872$, and 873 . Because of the relatively shallow depths of the sites studied, nannofloras are nicely to moderately preserved and the tiny and delicate species, which are prone to dissolution, are common. Gephyrocapsids are dominant and, at all sites, a gradual increase in abundance of the Gephyrocapsa group was observed from the lower to the upper Pleistocene (Figs. 1-4). Only in the uppermost Pleistocene, gephyrocapsids decrease in abundance and are progressively outnumbered by Emiliania huxleyi. In the lowermost Pleistocene reticulofenestrids are abundant, but this group becomes rare from the middle Pleistocene upward.

Within the genus Gephyrocapsa, different morphogroups show variable abundance through the Pleistocene, as pointed out by the gephyrocapsid composition illustrated in Figures 1 through 4. These changes in the proportions of gephyrocapsid taxa are extremely useful for biostratigraphy and are discussed below.

Pseudoemiliania lacunosa is generally more abundant in the lower than in the middle Pleistocene, and its progressive decline is paralleled by the increase in abundance of gephyrocapsids. However, at Site 880 , two abundance peaks of Pseudoemiliania lacunosa with values up to 


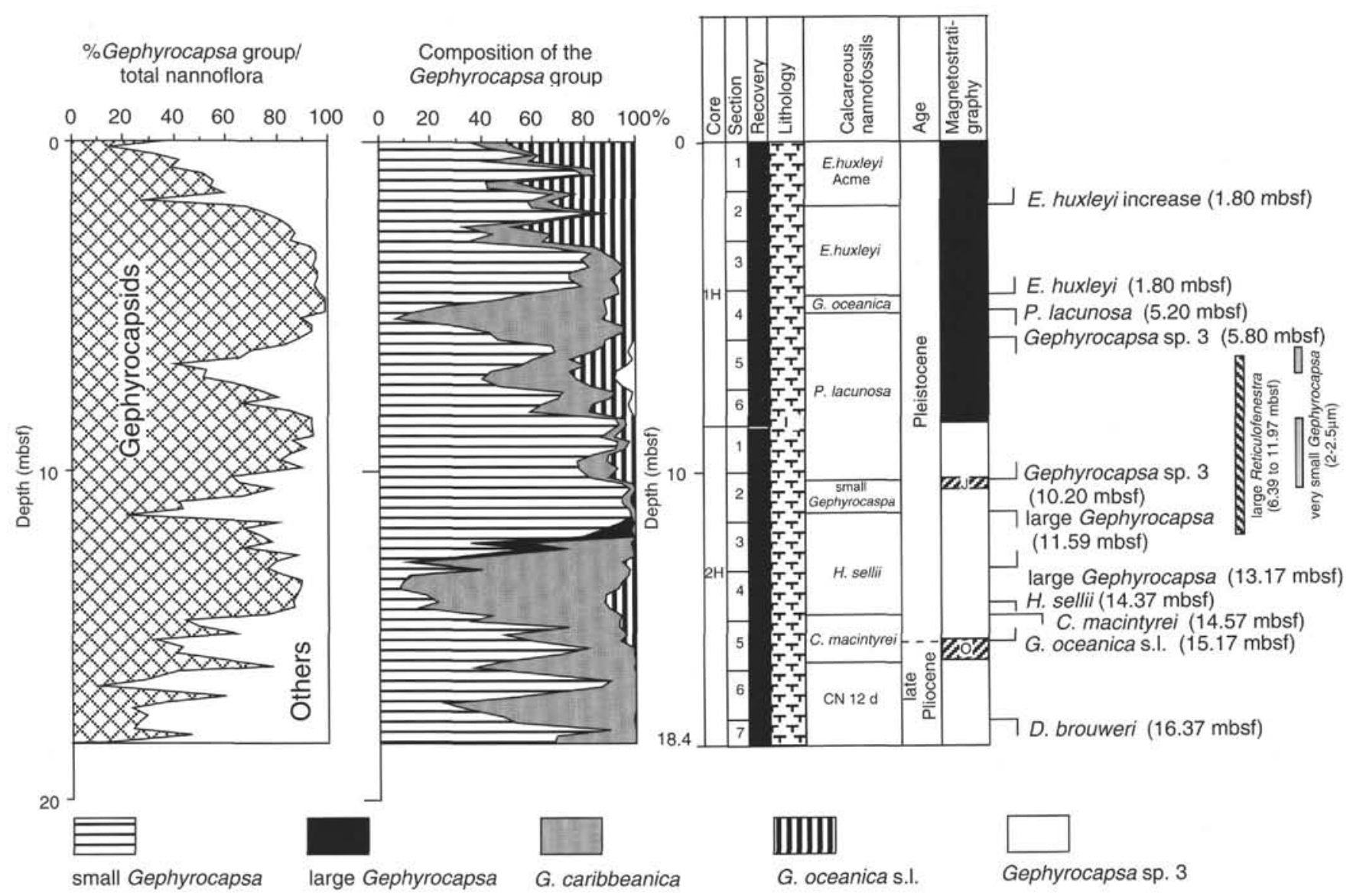

Figure 1. Abundance of gephyrocapsids vs. other nannofossils and composition (percentages) of various taxonomic classes within the Gephyrocapsa group at Site 880 (Takuyo-Daisan Guyot). On the right are the biostratigraphic results of quantitative analyses of nannofloras correlated with the magnetostratigraphy provided by Rack et al. (this volume). The Jaramillo and Olduvai subchrons are plotted in a diagonal pattern because they were tentatively recognized.

approximately $50 \%$ of the total nannoflora were detected in the middle Pleistocene. Also at Site 871, P. lacunosa shows an increase in abundance (approximately $25 \%$ of the total nannoflora) in the middle Pleistocene, and in coeval sediments at Site 872 this taxon increases to approximately $23 \%$ of the total nannoflora.

Several other nannofossil genera were observed, but they are usually represented by rare to few specimens. The complete nannofloral distribution in the Quaternary sequences at Sites 880, 871, 872, and 873 is reported in Tables 2 through 5.

\section{Site 880 (Takuyo-Daisan Guyot)}

The distribution of calcareous nannofossils in the Quaternary sequence at Site 880 is reported in Table 2. Percentages of biostratigraphically important taxa as well as those of the most abundant forms are plotted in Figure 5, and the abundance and composition of the gephyrocapsid group are illustrated in Figure 1.

Small and medium-sized Reticulofenestra and Pseudoemiliania lacunosa are abundant in the lower Pleistocene and both decline through the middle Pleistocene. Two abundance peaks of Pseudoemiliania lacunosa with values up to approximately $50 \%$ of the total nannoflora were detected in the middle Pleistocene, at 12.97 10.97 and $7.98-6.39$ mbsf. Also, the reticulofenestrids show a relative increase in abundance between 11.97 and 6.39 mbsf where they are represented exclusively by the large specimens.

Gephyrocapsids gradually increase in abundance upward and overwhelm over all the other nannofossils in the middle to upper Pleistocene, but markedly decrease in abundance in the uppermost Pleistocene, where they are outnumbered by Emiliania huxleyi. Within the
Gephyrocapsa group, the various taxa show marked changes in abundance (Fig. 1). The small Gephyrocapsa and G. caribbeanica dominate the lowermost Pleistocene, where G. oceanica s.l. appears and increases in abundance, but is never dominant. Between 13.17 and 11 . 59 mbsf, the large Gephyrocapsa spp. are common, but absent below or above this interval. The disappearance of the large Gephyrocapsa coincides with the base of dominant small Gephyrocapsa that constitute up to $97 \%$ of the gephyrocapsid group. In the interval immediately underlying the base of the small Gephyrocapsa Zone, the mediumsized specimens are virtually absent, whereas the large-sized ones are common.

Within the small gephyrocapsid class, specimens of 2 to $2.5 \mu \mathrm{m}$ in length become dominant at the base of the small Gephyrocapsa Zone (11.59-11.97 mbsf), in the lowermost part of the P. lacunosa Zone (8.40-10.54 mbsf), and in a short interval (6.19-6.59 mbsf) close to the end of the large Reticulofenestra interval.

At 10.20 mbsf, the medium-sized gephyrocapsids reenter the section and are represented by $G$. caribbeanica, G. oceanica s.l., and Gephyrocapsa sp. 3. The latter taxon was observed only between 10.20 and 5.80 mbsf, whereas both $G$. caribbeanica and $G$. oceanica s.l. occur in the remainder of the Pleistocene. A marked change in nannofloral composition was detected at 4.80 mbsf, where Emiliania huxleyi was first observed. At this level, G. caribbeanica sharply decrease in abundance, whereas the small specimens become dominant again within the Gephyrocapsa group.

Eleven biostratigraphic events and eight biozones were recognized at this site (Table 6); the depths of the nannofossil events are reported in Table 7 . The calibration of nannofossil biostratigraphy with magnetostratigraphy (Rack et al., this volume) is illustrated in 
Table 2. Quantitative distribution of Quaternary calcareous nannofossils at Site 880 (Takuyo-Daisan Guyot).

\begin{tabular}{|c|c|c|c|c|c|c|c|c|c|c|c|c|c|c|c|c|c|c|c|c|c|c|}
\hline $\begin{array}{l}\text { Core, section, } \\
\text { interval }(\mathrm{cm})\end{array}$ & $\begin{array}{r}\text { Depth } \\
\text { (mbsf) }\end{array}$ & 䓂 & 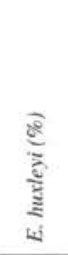 & 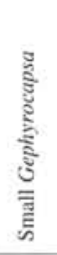 & 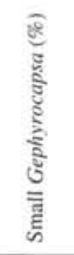 & 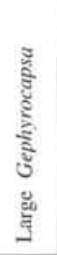 & 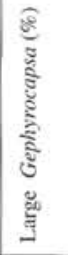 & $\begin{array}{l}\text { हैँ } \\
\text { हैँ } \\
\text { है } \\
\text { है }\end{array}$ & 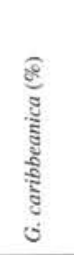 & 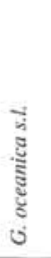 & 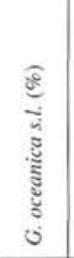 & 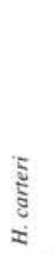 & 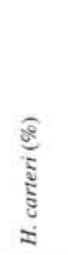 & $\frac{\text { है }}{\mathrm{s}}$ & 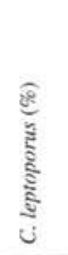 & نั & 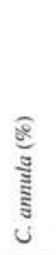 & 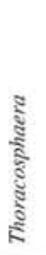 & 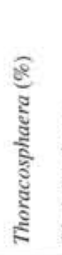 & 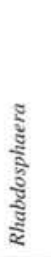 & 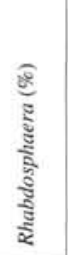 & 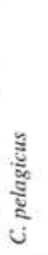 \\
\hline $44-880 \mathrm{~A}$ & & & & & & & & & & & & & & & & & & & & & & \\
\hline & 0.01 & 236 & 42.29 & 72 & 12.90 & 0 & 0.00 & 28 & 5.02 & 103 & 18.46 & 53 & 9.50 & 53 & 9.50 & 10 & 1.79 & 1 & 0.18 & 1 & 0.18 & 1 \\
\hline & 0.20 & & & 33 & & 0 & & 9 & 1.72 & 36 & 6.88 & 13 & 2.49 & 30 & 44 & 9 & 1.72 & 0 & 0.00 & 3 & 0 & 3 \\
\hline & 0 & 295 & 58 & 98 & 19 & 0 & 0 & 4 & 0.79 & 63 & 12.43 & 12 & 2 & 18 & & 7 & 38 & 0 & 00 & 6 & & 2 \\
\hline & 0 & 189 & 32 & 103 & 1 & 0 & & 41 & 6.95 & 101 & 17.12 & 82 & 13.90 & 53 & & 9 & .53 & 1 & 17 & 0 & & 9 \\
\hline 11 & 0.81 & 233 & 45 & 150 & 29 & 0 & & 15 & 2.94 & 34 & 6.65 & 46 & 9.00 & 18 & & 5 & .98 & 0 & 00 & 2 & & 8 \\
\hline 102 & 1.00 & 194 & 37 & 208 & 40.63 & 0 & 0.00 & 12 & 2.34 & 42 & 8.20 & 13 & 2.54 & 26 & 8 & 5 & 98 & 0 & 00 & 0 & & 12 \\
\hline it & 1.20 & 128 & 25 & 116 & 23,02 & 0 & & 33 & 6.55 & 129 & 25.60 & II & 2.18 & 70 & 13.89 & 12 & 38 & 0 & 00 & 0 & & 4 \\
\hline it & 1.40 & 135 & 26. & 116 & 22.79 & 0 & & 53 & 10.41 & 106 & 20.83 & 24 & 4.72 & 57 & 11.20 & 9 & .77 & 1 & 20 & 1 & & 2 \\
\hline $\mathrm{iH}$ & 1.60 & 105 & 20.47 & 206 & 40.16 & 0 & & 25 & 4.87 & 74 & 14.42 & 33 & 6.43 & 55 & 10.72 & 2 & 39 & i & 19 & i & & 7 \\
\hline & 1.80 & 282 & 54.76 & 82 & 15.92 & 0 & & 12 & 2.33 & 48 & 9.32 & 39 & 7.57 & 35 & 6.80 & 2 & 0.39 & 0 & .00 & 4 & & 6 \\
\hline $1 \mathrm{H}-2$ & 2.00 & 52 & 9.85 & 216 & 40.91 & 0 & & 44 & 8.33 & 100 & 18.94 & 42 & 7.95 & 57 & 10.80 & 9 & 1.70 & 0 & .00 & 0 & & 8 \\
\hline $\mathrm{IH}-2,6$ & 2.18 & 75 & 14.59 & 317 & 61.67 & 0 & 0 & 20 & 3.89 & 47 & 9.14 & 23 & 4.47 & is & 2.92 & 2 & 39 & 1 & .19 & 0 & & 6 \\
\hline iH- $2,90-92$ & 2.40 & 49 & 8.46 & 287 & 49.57 & 0 & & 28 & 4.84 & 155 & 26.77 & 1 & 0.17 & 40 & 6.91 & 0 & .00 & 0 & .00 & 0 & & 19 \\
\hline $1 \mathrm{H}-2,110-112$ & 2.60 & 23 & 4.5 & 142 & 28.17 & 0 & & 81 & 16.07 & 203 & 40.28 & 4 & 0.79 & 38 & 7.54 & 9 & .79 & 1 & 20 & 0 & & 2 \\
\hline $1 \mathrm{H}-2,130-132$ & 2.80 & 3 & 0.59 & 196 & 38.51 & 0 & & 103 & 20.24 & 144 & 28.29 & 9 & 1.77 & 42 & 8.25 & 3 & 59 & 0 & .00 & 0 & & 8 \\
\hline IH $-3,1-3$ & 3.01 & 13 & 2.5 & 150 & 29.47 & 0 & & 126 & 24.75 & 158 & 31.04 & 10 & 1.96 & 42 & 8.25 & I & .20 & 0 & .00 & 0 & & 7 \\
\hline $\mathrm{IH}-3,20-22$ & 3.20 & 13 & 2.30 & 354 & 62.65 & 0 & & 92 & 16.28 & 79 & 13.98 & 8 & 1.42 & 9 & 1.59 & 5 & 88 & 1 & 18 & 0 & & 3 \\
\hline $40-42$ & 3.40 & 12 & 2.3 & 396 & 78.26 & 0 & & 28 & 5 & 57 & 11.26 & 2 & & 5 & & 1 & 20 & 0 & 00 & 0 & & 5 \\
\hline if & & ii & 2.2 & 377 & 75.4 & 0 & & 62 & 12. & 38 & 7.60 & 2 & & 10 & & 0 & 00 & 0 & 00 & 0 & & 0 \\
\hline if & & 16 & 3.19 & 384 & 76.65 & 0 & & 63 & 12. & 24 & 4.79 & i & & iI & & 0 & e & 0 & & 0 & & 2 \\
\hline & & 8 & 1.5 & 357 & 70. & 0 & & 93 & 18 & 32 & 6.35 & 3 & & 8 & & 0 & & 1 & & 0 & & S \\
\hline & 4. & 13 & 2. & 357 & 70 & 0 & & 78 & & 47 & 9.33 & 2 & & 3 & & 0 & & 0 & & 1 & & 2 \\
\hline & & 17 & 3. & 372 & & ( & & 66 & & 35 & & 10 & & 0 & & 0 & & 0 & & 0 & & 1 \\
\hline & & 6 & 1.1 & & & & & 175 & & 33 & & 1 & & 5 & & 0 & & 1 & & 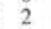 & & 2 \\
\hline & & 5 & i. & 228 & & & & & & 60 & 11 & 0 & & 0 & & 0 & & $\mathrm{i}$ & & & & i \\
\hline & & 0 & & 133 & 26 & & & & & 58 & & 0 & & & & 0 & & 0 & & & & 1 \\
\hline & & 0 & & 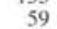 & iI & & & & & 57 & & 0 & & & & 0 & & 0 & & & & 0 \\
\hline & & 0 & & 8 & & & & & & 57 & & 3 & & 5 & & 0 & & 0 & & & & 0 \\
\hline & & 0 & & 149 & 29 & & & & & 23 & & 1 & & & & 0 & & 0 & & 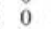 & & 0 \\
\hline & & 0 & & & & & & & & 21 & & 0 & & & & 0 & & 0 & & & & 0 \\
\hline & & 0 & & & & & & 186 & & 51 & 10 & 8 & & & & 0 & & 0 & & & & \\
\hline & & 0 & & & & & & 87 & & 44 & & 4 & & & & 0 & & 0 & & & & \\
\hline & & 0 & & & 47 & & & 52 & & 48 & & 7 & & & & 0 & & 0 & & & & \\
\hline & & 0 & & & 38 & & & 47 & & 81 & 15 & & & & & 0 & & 0 & & & & \\
\hline & & 0 & & & 22 & & & 52 & & 34 & 6. & & & & & 5 & & 1 & & & & 15 \\
\hline & & 0 & & & 22 & & & 87 & 16 & 51 & 9. & & & & & 9 & & i & & & & \\
\hline & & 0 & 0 & & 20 & & & 99 & 18 & & & & & & & & & 0 & & & & \\
\hline & & 0 & & & 30. & 0 & & 125 & & 44 & 8 & & & & & & & 0 & & & & \\
\hline & & 0 & & & & 0 & & 78 & & 25 & 4. & 1 & & & & 10 & & 0 & & & & \\
\hline & & 0 & & & & 0 & & 85 & & 35 & 6. & 11 & & 21 & & 8 & & 1 & & & & 5 \\
\hline & & 0 & & & & 0 & & 67 & & 47 & 9. & 7 & & . & & 5 & & 0 & & & & 0 \\
\hline & & 0 & & & & 0 & & 101 & 19. & 69 & 13.5 & 2 & & ( & & 22 & & 0 & & 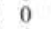 & & 6 \\
\hline & & 0 & & & & ( & & 16 & 3 & 19 & 3.7 & 2 & & 6 & & & & I & & 4 & & 9 \\
\hline & & 0 & & & & ( & & 12 & & 15 & & 2 & & 3 & & 7 & & 0 & & 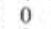 & & 0 \\
\hline & & 0 & & & & i & & 24 & 4 & 38 & 7. & 0 & & 13 & & 2 & & 0 & & 0 & & 1 \\
\hline & & 0 & & & & 0 & & 18 & 3 & 9 & 1. & 4 & & 16 & & 3 & & 0 & & & & 1 \\
\hline & & 0 & & & & 0 & & 16 & & 19 & 3. & 5 & & r & & 2 & & 0 & & 1 & & 2 \\
\hline & & 0 & & & & 0 & & 26 & & 44 & 8. & 4 & & 18 & & 2 & & 0 & & i & & 1 \\
\hline & & 0 & & & & 0 & & 54 & & 7 & & 4 & & 28 & & $i$ & & 0 & & 0 & & 4 \\
\hline & & 0 & & & & 0 & & 0 & 13 & 31 & & 2 & & 20 & & 3 & & 0 & & 0 & & 14 \\
\hline & & 0 & & & & 0 & & 25 & & 31 & $\begin{array}{l}0 . \\
6 .\end{array}$ & 2 & & 24 & & 2 & & $\begin{array}{l}0 \\
0\end{array}$ & & 0 & & 7 \\
\hline & & 0 & & & & 0 & & 13 & & 5 & & 2 & & 2 & & 4 & & 0 & & 0 & & 0 \\
\hline & & 0 & & & & 0 & & 13 & & i & & 8 & & 33 & & 1 & & 0 & & 0 & & 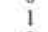 \\
\hline & & 0 & & & & 0 & & 11 & & 7 & & 5 & & 0 & & i & & 1 & & 0 & & 2 \\
\hline & & & & & & 0 & & $\because$ & & 4 & & $i$ & & & & 3 & & 0 & & 0 & & $i$ \\
\hline & & & & & & a & & 2 & & i & & 4 & & & & 0 & & 0 & & 0 & & 4 \\
\hline & & & & & & & & 4 & & 0 & & 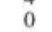 & & i & 14. & To & & 0 & & 0 & & 7 \\
\hline & & & & & & & & $\approx$ & & 5 & & & & 9 & & & & $i$ & & 2 & & 3 \\
\hline & & & & & & & & 5 & & 1 & & & & & & 2 & & 0 & & $i$ & & 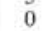 \\
\hline & & & & & & & & $i$ & & 2 & & & & & & 1 & & 0 & & 0 & & \\
\hline & & & & & & & & 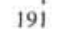 & & & & & & & & i & & 0 & & $\begin{array}{l}y_{0} \\
0\end{array}$ & & 2 \\
\hline & & 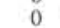 & & & 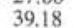 & & & & & & & 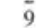 & & & & i & & 0 & & 0 & & 4 \\
\hline & & & & is & 28 & & & & & & & & & & & & & & & 0 & & 3 \\
\hline & & & & & & & & & & & & & & & & & & 0 & & 0 & & 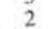 \\
\hline & & & & 147 & 28 & & & & & & & & & & & & & & & i & & 1 \\
\hline & & & & 4 & & & & & & & & & & & & & & & & 0 & & \\
\hline & & & & & & & & & & & & & & & & & & & & 0 & & \\
\hline & & & & 3 & & & & & & & & & & & & & & & & 0 & & \\
\hline & & & & & & & & & & & & & & & & & & & & & & \\
\hline & & & & & & & & & & & & & & & & & & & & & & \\
\hline & & & & & & & & & & & & & & & & & & & & & & \\
\hline & & & & & & & & & & & & & & & & & & & & & & \\
\hline & & & & & & & & & & & & & & & & & & & & & & \\
\hline & & & & & & & & & & & & & & & & & & & & & & \\
\hline
\end{tabular}


Table 2 (continued).

\begin{tabular}{|c|c|c|c|c|c|c|c|c|c|c|c|c|c|c|c|c|c|c|c|c|c|c|c|}
\hline 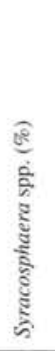 & $\frac{\vdots}{3}$ & $\frac{\mathbb{8}}{\sqrt[6]{3}}$ & 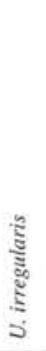 & 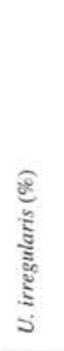 & 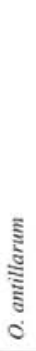 & 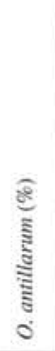 & 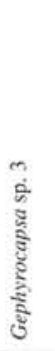 & 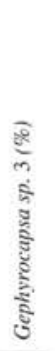 & $\begin{array}{l}\equiv \\
\vdots \\
\vdots\end{array}$ & 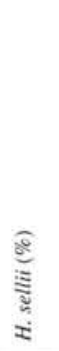 & 离 & 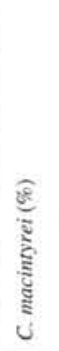 & 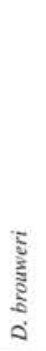 & 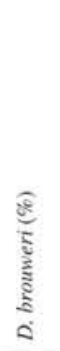 & 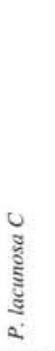 & 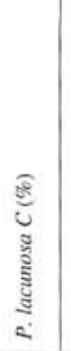 & 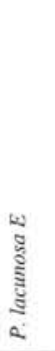 & 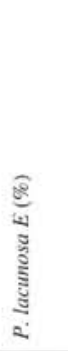 & 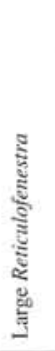 & 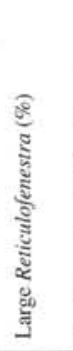 & 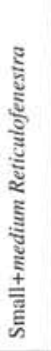 & 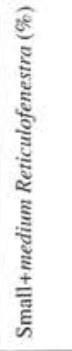 & 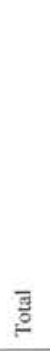 \\
\hline 0.00 & 0 & 0.00 & 0 & 0.00 & 0 & 0.00 & 0 & 0.00 & 0 & 0.00 & 0 & 0.00 & 0 & 0.00 & 0 & 0.00 & 0 & 0.00 & 0 & 0.00 & 0 & 0.00 & 558 \\
\hline 0.76 & I & 0.19 & 2 & 0.38 & 0 & 0.00 & 0 & 0.00 & 0 & 0.00 & 0 & 0.00 & 0 & 0.00 & 0 & 0.00 & 0 & 0.00 & 0 & 0.00 & 0 & 0.00 & 523 \\
\hline 0.20 & 0 & 0.00 & 1 & 0.20 & 0 & 0.00 & 0 & 0.00 & 0 & 0.00 & 0 & 0.00 & 0 & 0.00 & 0 & 0.00 & 0 & 0.00 & 0 & 0.00 & 0 & 0.00 & 507 \\
\hline 0.00 & 0 & 0.00 & 2 & 0.34 & 0 & 0.00 & 0 & 0.00 & 0 & 0.00 & 0 & 0.00 & 0 & 0.00 & 0 & 0.00 & 0 & 0.00 & 0 & 0.00 & 0 & 0.00 & 590 \\
\hline 0.00 & 0 & 0.00 & 0 & 0.00 & 0 & 0.00 & 0 & 0.00 & 0 & 0.00 & 0 & 0.00 & 0 & 0.00 & 0 & 0.00 & 0 & 0.00 & 0 & 0.00 & 0 & 0.00 & 511 \\
\hline 0.00 & 0 & 0.00 & 0 & 0.00 & 0 & 0.00 & 0 & 0.00 & 0 & 0.00 & 0 & 0.00 & 0 & 0.00 & 0 & 0.00 & 0 & 0.00 & 0 & 0.00 & 0 & 0.00 & 512 \\
\hline 0.20 & 0 & 0.00 & 0 & 0.00 & 0 & 0.00 & 0 & 0.00 & 0 & 0.00 & 0 & 0.00 & 0 & 0.00 & 0 & 0.00 & 0 & 0.00 & 0 & 0.00 & 0 & 0.00 & 504 \\
\hline 0.20 & 1 & 0.20 & 3 & 0.59 & 0 & 0.00 & 0 & 0.00 & 0 & 0.00 & 0 & 0.00 & 0 & 0.00 & 0 & 0.00 & 0 & 0.00 & 0 & 0.00 & 0 & 0.00 & 509 \\
\hline 0.58 & 0 & 0.00 & 1 & 0.19 & 0 & 0.00 & 0 & 0.00 & 0 & 0.00 & 0 & 0.00 & 0 & 0.00 & 0 & 0.00 & 0 & 0.00 & 0 & 0.00 & 0 & 0.00 & 513 \\
\hline 0.19 & 0 & 0.00 & 2 & 0.39 & 2 & 0.39 & 0 & 0.00 & 0 & 0.00 & 0 & 0.00 & 0 & 0.00 & 0 & 0.00 & 0 & 0.00 & 0 & 0.00 & 0 & 0.00 & 515 \\
\hline 0.00 & 0 & 0.00 & 0 & 0.00 & 0 & 0.00 & 0 & 0.00 & 0 & 0.00 & 0 & 0.00 & 0 & 0.00 & 0 & 0.00 & 0 & 0.00 & 0 & 0.00 & 0 & 0.00 & 528 \\
\hline 0.97 & 2 & 0.39 & 1 & 0.19 & 0 & 0.00 & 0 & 0.00 & 0 & 0.00 & 0 & 0.00 & 0 & 0.00 & 0 & 0.00 & 0 & 0.00 & 0 & 0.00 & 0 & 0.00 & 514 \\
\hline 0.00 & 0 & 0.00 & 0 & 0.00 & 0 & 0.00 & 0 & 0.00 & 0 & 0.00 & 0 & 0.00 & 0 & 0.00 & 0 & 0.00 & 0 & 0.00 & 0 & 0.00 & 0 & 0.00 & 579 \\
\hline 0.00 & 0 & 0.00 & 1 & 0.20 & 0 & 0.00 & 0 & 0.00 & 0 & 0.00 & 0 & 0.00 & 0 & 0.00 & 0 & 0.00 & 0 & 0.00 & 0 & 0.00 & 0 & 0.00 & 504 \\
\hline 0.20 & 0 & 0.00 & 0 & 0.00 & 0 & 0.00 & 0 & 0.00 & 0 & 0.00 & 0 & 0.00 & 0 & 0.00 & 0 & 0.00 & 0 & 0.00 & 0 & 0.00 & 0 & 0.00 & 509 \\
\hline 0.00 & 0 & 0.00 & 2 & 0.39 & 0 & 0.00 & 0 & 0.00 & 0 & 0.00 & 0 & 0.00 & 0 & 0.00 & 0 & 0.00 & 0 & 0.00 & 0 & 0.00 & 0 & 0.00 & 509 \\
\hline 0.00 & 1 & 0.18 & 0 & 0.00 & 0 & 0.00 & 0 & 0.00 & 0 & 0.00 & 0 & 0.00 & 0 & 0.00 & 0 & 0.00 & 0 & 0.00 & 0 & 0.00 & 0 & 0.00 & 565 \\
\hline 0.00 & 0 & 0.00 & 0 & 0.00 & 0 & 0.00 & 0 & 0.00 & 0 & 0.00 & 0 & 0.00 & 0 & 0.00 & 0 & 0.00 & 0 & 0.00 & 0 & 0.00 & 0 & 0.00 & 506 \\
\hline 0.00 & 0 & 0.00 & 0 & 0.00 & 0 & 0.00 & 0 & 0.00 & 0 & 0.00 & 0 & 0.00 & 0 & 0.00 & 0 & 0.00 & 0 & 0.00 & 0 & 0.00 & 0 & 0.00 & 500 \\
\hline 0.00 & 0 & 0.00 & 0 & 0.00 & 0 & 0.00 & 0 & 0.00 & 0 & 0.00 & 0 & 0.00 & 0 & 0.00 & 0 & 0.00 & 0 & 0.00 & 0 & 0.00 & 0 & 0.00 & 501 \\
\hline 0.20 & 0 & 0.00 & 0 & 0.00 & 0 & 0.00 & 0 & 0.00 & 0 & 0.00 & 0 & 0.00 & 0 & 0.00 & 0 & 0.00 & 0 & 0.00 & 0 & 0.00 & 0 & 0.00 & 504 \\
\hline 0.20 & 0 & 0.00 & 0 & 0.00 & 0 & 0.00 & 0 & 0.00 & 0 & 0.00 & 0 & 0.00 & 0 & 0.00 & 0 & 0.00 & 0 & 0.00 & 0 & 0.00 & 0 & 0.00 & 504 \\
\hline 0.00 & 0 & 0.00 & 0 & 0.00 & 0 & 0.00 & 0 & 0.00 & 0 & 0.00 & 0 & 0.00 & 0 & 0.00 & 0 & 0.00 & 0 & 0.00 & 0 & 0.00 & 0 & 0.00 & 501 \\
\hline 0.20 & 0 & 0.00 & 0 & 0.00 & 0 & 0.00 & 0 & 0.00 & 0 & 0.00 & 0 & 0.00 & 0 & 0.00 & 0 & 0.00 & 0 & 0.00 & 0 & 0.00 & 0 & 0.00 & 507 \\
\hline 0.20 & 0 & 0.00 & 0 & 0.00 & 0 & 0.00 & 0 & 0.00 & 0 & 0.00 & 0 & 0.00 & 0 & 0.00 & 0 & 0.00 & 0 & 0.00 & 0 & 0.00 & 0 & 0.00 & 502 \\
\hline 0.00 & 0 & 0.00 & 0 & 0.00 & 0 & 0.00 & 0 & 0.00 & 0 & 0.00 & 0 & 0.00 & 0 & 0.00 & 3 & 0.60 & 1 & 0.20 & 0 & 0.00 & 0 & 0.00 & 500 \\
\hline 0.00 & 0 & 0.00 & 0 & 0.00 & 0 & 0.00 & 0 & 0.00 & 0 & 0.00 & 0 & 0.00 & 0 & 0.00 & 1 & 0.20 & 5 & 0.99 & 0 & 0.00 & 0 & 0.00 & 504 \\
\hline 0.00 & 0 & 0.00 & 0 & 0.00 & 0 & 0.00 & 0 & 0.00 & 0 & 0.00 & 0 & 0.00 & 0 & 0,00 & 20 & 3.98 & 15 & 2.99 & 0 & 0.00 & 0 & 0.00 & 502 \\
\hline 0.00 & 0 & 0.00 & 0 & 0.00 & 0 & 0.00 & 0 & 0.00 & 0 & 0.00 & 0 & 0.00 & 0 & 0.00 & 17 & 3.40 & 12 & 2.40 & 0 & 0.00 & 0 & 0.00 & 500 \\
\hline 0.00 & 0 & 0.00 & 0 & 0.00 & 0 & 0.00 & 3 & 0.60 & 0 & 0.00 & 0 & 0.00 & 0 & 0.00 & 5 & 1.00 & 17 & 3.39 & 0 & 0.00 & 0 & 0.00 & 502 \\
\hline 0.00 & 0 & 0.00 & 0 & 0.00 & 0 & 0.00 & 3 & 0.59 & 0 & 0.00 & 0 & 0.00 & 0 & 0.00 & 14 & 2.77 & 25 & 4.94 & 0 & 0.00 & 0 & 0.00 & 506 \\
\hline 0.00 & 0 & 0.00 & 0 & 0.00 & 0 & 0.00 & 8 & 1.57 & 0 & 0.00 & 0 & 0.00 & 0 & 0.00 & 19 & 3.73 & 44 & 8.63 & 0 & 0.00 & 0 & 0.00 & 510 \\
\hline 0.00 & 0 & 0.00 & 0 & 0.00 & 0 & 0.00 & 12 & 2.34 & 0 & 0.00 & 0 & 0.00 & 0 & 0.00 & 22 & 4.29 & 112 & 21.83 & 10 & 1.95 & 10 & 1.95 & 513 \\
\hline 0.00 & 0 & 0.00 & 0 & 0.00 & 0 & 0.00 & 5 & 0.98 & 0 & 0.00 & 0 & 0.00 & 0 & 0.00 & 12 & 2.35 & 123 & 24.07 & 5 & 0.98 & 5 & 0.98 & 511 \\
\hline 0.38 & I & 0.19 & 0 & 0.00 & 0 & 0.00 & 8 & 1.52 & 0 & 0.00 & 0 & 0.00 & 0 & 0.00 & 26 & 4.95 & 224 & 42.67 & 0 & 0.00 & 0 & 0.00 & 525 \\
\hline 0.37 & 2 & 0.37 & 0 & 0.00 & 0 & 0.00 & 21 & 3.92 & 0 & 0.00 & 0 & 0.00 & 0 & 0.00 & 20 & 3.73 & 176 & 32.84 & 0 & 0.00 & 0 & 0.00 & 536 \\
\hline 0.00 & 0 & 0.00 & 0 & 0.00 & 0 & 0.00 & 23 & 4.35 & 0 & 0.00 & 0 & 0.00 & 0 & 0.00 & 26 & 4.91 & 162 & 30.62 & 0 & 0.00 & 0 & 0.00 & 529 \\
\hline 0.00 & 0 & 0.00 & 0 & 0.00 & 0 & 0.00 & 18 & 3.41 & 1 & 0.19 & 0 & 0.00 & 0 & 0.00 & 12 & 2.27 & 81 & 15.34 & 0 & 0.00 & 0 & 0.00 & 528 \\
\hline 0.19 & 0 & 0.00 & 0 & 0.00 & 0 & 0.00 & 6 & 1.15 & 0 & 0.00 & 0 & 0.00 & 0 & 0.00 & 15 & 2.88 & 79 & 15.16 & 0 & 0.00 & 0 & 0.00 & 521 \\
\hline 0.00 & 0 & 0.00 & 0 & 0.00 & 0 & 0.00 & 8 & 1.51 & 0 & 0.00 & 0 & 0.00 & 0 & 0.00 & 1 & 0.19 & 47 & 8.88 & 0 & 0.00 & 0 & 0.00 & 529 \\
\hline 0.00 & 0 & 0.00 & 0 & 0.00 & 0 & 0.00 & 10 & 1.96 & 0 & 0.00 & 0 & 0.00 & 0 & 0.00 & 15 & 2.94 & 111 & 21.72 & 33 & 6.46 & 33 & 6.46 & 511 \\
\hline 0.00 & 0 & 0.00 & 0 & 0.00 & 0 & 0.00 & 9 & 1.77 & 0 & 0.00 & 0 & 0.00 & 0 & 0.00 & 6 & 1.18 & 38 & 7.48 & 3 & 0.59 & 3 & 0.59 & 508 \\
\hline 0.00 & 0 & 0.00 & 0 & 0.00 & 0 & 0.00 & 2 & 0.39 & 0 & 0.00 & 0 & 0.00 & 0 & 0.00 & 2 & 0.39 & 6 & 1.18 & 0 & 0.00 & 0 & 0.00 & 509 \\
\hline 0.00 & 0 & 0.00 & 0 & 0.00 & 0 & 0.00 & 3 & 0.60 & 0 & 0.00 & 0 & 0.00 & 0 & 0.00 & 10 & 1.98 & 10 & 1.98 & 0 & 0.00 & 0 & 0.00 & 504 \\
\hline 0.00 & 0 & 0.00 & 0 & 0.00 & 0 & 0.00 & 1 & 0.20 & 0 & 0.00 & 0 & 0.00 & 0 & 0.00 & 2 & 0.40 & 13 & 2.59 & 0 & 0.00 & 0 & 0.00 & 502 \\
\hline 0.00 & 1 & 0.20 & 0 & 0.00 & 0 & 0.00 & 3 & 0.59 & 0 & 0.00 & 0 & 0.00 & 0 & 0.00 & 12 & 2.36 & 34 & 6.69 & 0 & 0.00 & 0 & 0.00 & 508 \\
\hline 0.00 & 0 & 0.00 & 0 & 0.00 & 0 & 0.00 & 1 & 0.20 & 0 & 0.00 & 0 & 0.00 & 0 & 0.00 & 6 & 1.18 & 16 & 3.16 & 5 & 0.99 & 5 & 0.99 & 507 \\
\hline 0.20 & 0 & 0.00 & 0 & 0.00 & 0 & 0.00 & 2 & 0.40 & 0 & 0.00 & 0 & 0.00 & 0 & 0.00 & 8 & 1.59 & 37 & 7.37 & 5 & 1.00 & 5 & 1.00 & 502 \\
\hline 0.00 & 0 & 0.00 & 0 & 0.00 & 0 & 0.00 & 2 & 0.36 & 0 & 0.00 & 0 & 0.00 & 0 & 0.00 & 15 & 2.71 & 48 & 8.68 & 9 & 1.63 & 9 & 1.63 & 553 \\
\hline 0.00 & 0 & 0.00 & 0 & 0.00 & 0 & 0.00 & 3 & 0.58 & 0 & 0.00 & 0 & 0.00 & 0 & 0.00 & 7 & 1.36 & 12 & 2.33 & 10 & 1.94 & 10 & 1.94 & 516 \\
\hline 0.00 & 0 & 0.00 & 0 & 0.00 & 0 & 0.00 & 2 & 0.40 & 0 & 0.00 & 0 & 0.00 & 0 & 0.00 & 16 & 3.16 & 49 & 9.68 & 92 & 18.18 & 92 & 18.18 & 506 \\
\hline 0.00 & 0 & 0.00 & 0 & 0.00 & 0 & 0.00 & 0 & 0.00 & 0 & 0.00 & 0 & 0.00 & 0 & 0.00 & 11 & 2.17 & 35 & 6.90 & 103 & 20.32 & 103 & 20.32 & 507 \\
\hline 0.00 & 0 & 0.00 & 0 & 0.00 & 0 & 0.00 & 0 & 0.00 & 0 & 0.00 & 0 & 0.00 & 0 & 0.00 & 7 & 1.39 & 39 & 7.75 & 23 & 4.57 & 23 & 4.57 & 503 \\
\hline 0.00 & 0 & 0.00 & 0 & 0.00 & 0 & 0.00 & 0 & 0.00 & 0 & 0.00 & 0 & 0.00 & 0 & 0.00 & 6 & 1.19 & 48 & 9.49 & 97 & 19.17 & 97 & 19.17 & 506 \\
\hline 0.00 & 0 & 0.00 & 0 & 0.00 & 0 & 0.00 & 0 & 0.00 & 0 & 0.00 & 0 & 0.00 & 0 & 0.00 & 23 & 4.56 & 131 & 25.99 & 93 & 18.45 & 93 & 18.45 & 504 \\
\hline 0.00 & 0 & 0.00 & 0 & 0.00 & 0 & 0.00 & 0 & 0.00 & 0 & 0.00 & 0 & 0.00 & 0 & 0.00 & 12 & 2.33 & 153 & 29.77 & 81 & 15.76 & 81 & 15.76 & 514 \\
\hline 0.00 & 0 & 0.00 & 0 & 0.00 & 0 & 0.00 & 0 & 0.00 & 0 & 0.00 & 0 & 0.00 & 0 & 0.00 & 13 & 2.56 & 265 & 52.27 & 48 & 9.47 & 48 & 9.47 & 507 \\
\hline 0.00 & 0 & 0.00 & 0 & 0.00 & 0 & 0.00 & 0 & 0.00 & 0 & 0.00 & 0 & 0.00 & 0 & 0.00 & 5 & 0.94 & 77 & 14.53 & 6 & 1.13 & 6 & 1.13 & 530 \\
\hline 0.00 & 0 & 0.00 & 0 & 0.00 & 0 & 0.00 & 0 & 0.00 & 0 & 0.00 & 0 & 0.00 & 0 & 0.00 & 22 & 4.05 & 136 & 25.05 & 1 & 0.18 & 1 & 0.18 & 543 \\
\hline 0.00 & 0 & 0.00 & 0 & 0.00 & 0 & 0.00 & 0 & 0.00 & 0 & 0.00 & 0 & 0.00 & 0 & 0.00 & 101 & 17.06 & 12 & 2.03 & i & 0.17 & 1 & 0.17 & 592 \\
\hline 0.00 & 0 & 0.00 & 0 & 0.00 & 0 & 0.00 & 0 & 0.00 & 0 & 0.00 & 0 & 0.00 & 0 & 0.00 & 4 & 0.66 & 92 & 15,16 & 0 & 0.00 & 0 & 0.00 & 607 \\
\hline 0.00 & 0 & 0.00 & 0 & 0.00 & 0 & 0.00 & 0 & 0.00 & 1 & 0.15 & 0 & 0.00 & 0 & 0.00 & $i$ & 0.15 & 49 & 7.47 & 0 & 0.00 & 0 & 0.00 & 656 \\
\hline 0.00 & 0 & 0.00 & 0 & 0.00 & 0 & 0.00 & 0 & 0.00 & 0 & 0.00 & 0 & 0.00 & 0 & 0.00 & i & 0.18 & 40 & 7.33 & 0 & 0.00 & 0 & 0.00 & 546 \\
\hline 0.00 & 0 & 0.00 & 0 & 0.00 & 0 & 0.00 & 0 & 0.00 & 0 & 0.00 & 0 & 0.00 & 0 & 0.00 & 6 & 1.17 & 60 & 11.72 & 0 & 0.00 & 0 & 0.00 & 512 \\
\hline 0.00 & 0 & 0.00 & 0 & 0.00 & 0 & 0.00 & 0 & 0.00 & 0 & 0.00 & 0 & 0.00 & 0 & 0.00 & 3 & 0.59 & 88 & 17.19 & 0 & 0.00 & 0 & 0.00 & 512 \\
\hline 0.00 & 0 & 0.00 & 0 & 0.00 & 0 & 0.00 & 0 & 0.00 & 0 & 0.00 & 0 & 0.00 & 0 & 0.00 & 4 & 0.78 & 47 & 9.13 & 0 & 0.00 & 0 & 0.00 & 515 \\
\hline 0.40 & 0 & 0.00 & 0 & 0.00 & 0 & 0.00 & 0 & 0.00 & 0 & 0.00 & 0 & 0.00 & 0 & 0.00 & 1 & 0.20 & 19 & 3.75 & 0 & 0.00 & 0 & 0.00 & 506 \\
\hline 0.20 & 0 & 0.00 & 0 & 0.00 & 0 & 0.00 & 0 & 0.00 & 0 & 0.00 & 0 & 0.00 & 0 & 0.00 & 3 & 0.60 & 31 & 6.16 & 0 & 0.00 & 0 & 0.00 & 503 \\
\hline 0.40 & 0 & 0.00 & 0 & 0.00 & 0 & 0.00 & 0 & 0.00 & 0 & 0.00 & 0 & 0.00 & 0 & 0.00 & 4 & 0.79 & 21 & 4.15 & 0 & 0.00 & 0 & 0.00 & 506 \\
\hline 0.39 & 0 & 0.00 & 0 & 0.00 & 2 & 0.39 & 0 & 0.00 & 0 & 0.00 & 0 & 0.00 & 0 & 0.00 & 5 & 0.98 & 29 & 5.69 & 0 & 0.00 & 0 & 0.00 & 510 \\
\hline 0.00 & 0 & 0.00 & 0 & 0.00 & 0 & 0.00 & 0 & 0.00 & 0 & 0.00 & 0 & 0.00 & 0 & 0.00 & 3 & 0.60 & 41 & 8.17 & 0 & 0.00 & 0 & 0.00 & 502 \\
\hline 0.00 & 0 & 0.00 & 0 & 0.00 & 0 & 0.00 & 0 & 0.00 & 1 & 0.20 & 0 & 0.00 & 0 & 0.00 & 2 & 0.39 & 37 & 7.30 & 0 & 0.00 & 37 & 7.30 & 507 \\
\hline 0.00 & 0 & 0.00 & 0 & 0.00 & 0 & 0.00 & 0 & 0.00 & 0 & 0.00 & 4 & 0.79 & 0 & 0.00 & 10 & 1.97 & 75 & 14.76 & 0 & 0.00 & 139 & 27.36 & 508 \\
\hline 0.00 & 0 & 0.00 & 0 & 0.00 & 0 & 0.00 & 0 & 0.00 & 0 & 0.00 & 6 & 1.18 & 0 & 0.00 & 17 & 3.35 & 170 & 33.46 & 0 & 0.00 & 30 & 5.91 & 508 \\
\hline
\end{tabular}


Table 2 (continued).

\begin{tabular}{|c|c|c|c|c|c|c|c|c|c|c|c|c|c|c|c|c|c|c|c|c|c|c|c|c|}
\hline $\begin{array}{l}\text { Core, section, } \\
\text { interval }(\mathrm{cm})\end{array}$ & $\begin{array}{l}\text { Depth } \\
\text { (mbsf) }\end{array}$ & 胥 & $\frac{8}{3}$ & 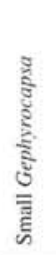 & 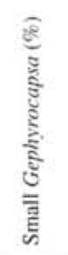 & 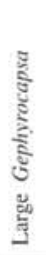 & 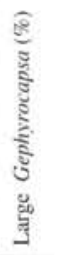 & 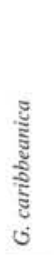 & 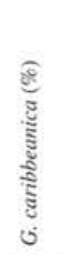 & 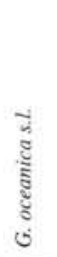 & 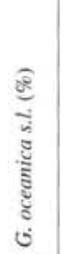 & $\begin{array}{l}\text { हूँ } \\
\text { हू } \\
\text { I }\end{array}$ & 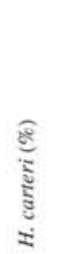 & $\frac{3}{\frac{n}{2}}$ & 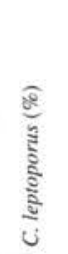 & 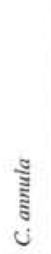 & 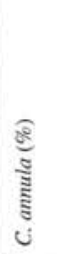 & $\begin{array}{l}\text { हूँ } \\
\text { हूँ } \\
\text { हूँ } \\
\text { हू }\end{array}$ & 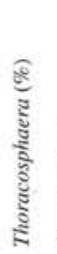 & 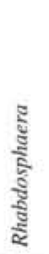 & 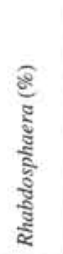 & 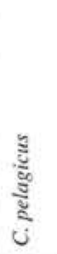 & 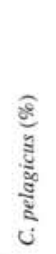 & 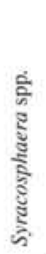 \\
\hline $2 \mathrm{H}-5,20-22$ & 14.97 & 0 & 0.00 & 157 & 31.03 & 0 & 0.00 & 155 & 30.63 & 12 & 2.37 & 8 & 1.58 & 28 & 5.53 & 0 & 0.00 & 0 & 0.00 & 0 & 0.00 & 9 & 1.78 & 0 \\
\hline $2 \mathrm{H}-5$. & 15. & 0 & & 102 & 19.54 & 0 & 0.00 & 58 & 11.11 & 10 & 1.92 & 2 & 0.38 & 26 & 4.98 & 0 & 0.00 & 0 & 0.00 & 0 & 0.00 & 13 & 2.49 & 0 \\
\hline $2 \mathrm{H}-5,62-64$ & 15.39 & 0 & 0.00 & 180 & 34.88 & 0 & 0.00 & 40 & 7.75 & 2 & 0.39 & 1 & 0.19 & 36 & 6.98 & 0 & 0.00 & 0 & 0.00 & 0 & 0.00 & 3 & 0.58 & 0 \\
\hline $2 \mathrm{H}-5,80-82$ & 15.57 & 0 & 0.00 & 150 & 28.74 & 0 & 0.00 & 60 & 11.49 & 2 & 0.38 & 11 & 2.11 & 35 & 6.70 & 0 & 0.00 & 0 & 0.00 & 0 & 0.00 & 5 & 0.96 & 1 \\
\hline $2 \mathrm{H}-5.100-102$ & 15.77 & 0 & 0.00 & 162 & 28.98 & 0 & 0.00 & 180 & 32.20 & 0 & 0.00 & 5 & 0.89 & 25 & 4.47 & 1 & 0.18 & 0 & 0.00 & 0 & & 10 & 1.79 & 1 \\
\hline $2 \mathrm{H}-5,120-122$ & 15.97 & 0 & 0.00 & 150 & 29.01 & 0 & 0.00 & 253 & 48.94 & 2 & 0.39 & 1 & 0.19 & 5 & 0.97 & 0 & 0.00 & 0 & 0.00 & 0 & & 5 & 0.97 & 0 \\
\hline $2 \mathrm{H}-5,136-138$ & 16.13 & 0 & 0.00 & 118 & 22.96 & 0 & 0.00 & 93 & 18.09 & 0 & 0.00 & 8 & 1.56 & 28 & 5.45 & 0 & 0.00 & 0 & 0.00 & 0 & & 0 & 0.00 & 0 \\
\hline $2 \mathrm{H}-6,1-12$ & 16.37 & 0 & 0.00 & 135 & 25.76 & 0 & 0.00 & is & 2.8 & 0 & 0.00 & 8 & 1.53 & 45 & 8.59 & 2 & 0.38 & 0 & 0.00 & 0 & & 14 & 2.67 & 0 \\
\hline $2 \mathrm{H}-6,30-32$ & 16.57 & 0 & 0.00 & 50 & 9.42 & 0 & 0.00 & 8 & 1.51 & 0 & 0.00 & 1 & 0.19 & 77 & 14.50 & 3 & 0.56 & 0 & 0.00 & 0 & & 11 & 2.07 & 3 \\
\hline $2 \mathrm{H}-6,60-62$ & 16.87 & 0 & 0.00 & 163 & 31.05 & 0 & 0.00 & 151 & 28.76 & 0 & 0.00 & 18 & & 28 & 5.33 & 0 & 0.00 & 0 & 0.00 & 0 & & 12 & 2.29 & $i$ \\
\hline $2 \mathrm{H}-6,81-83$ & 17,08 & 0 & & 50 & & 0 & 0.00 & 144 & 27,02 & 0 & 0.00 & 1 & & 62 & 11.63 & 0 & 0.00 & 0 & 0.00 & 0 & & 14 & 2.63 & 0 \\
\hline $2 \mathrm{H}-6.97-99$ & 17.24 & 0 & 0.00 & 42 & & 0 & 0.00 & 78 & 15.00 & 0 & 0.00 & i & & 55 & 10.58 & 0 & 0.00 & 0 & 0.00 & 0 & & 5 & 0.96 & i \\
\hline $2 \mathrm{H}-6,120-122$ & 17.47 & 0 & 0. & 78 & 14.08 & 0 & 0.00 & 83 & 14.98 & i & 0.18 & 3 & 0.54 & 38 & & 0 & 0.00 & 0 & 0.00 & 0 & 0.00 & 5 & 0.90 & 0 \\
\hline $2 \mathrm{H}-6,139-141$ & 17.66 & 0 & 0.00 & 73 & 13.75 & 0 & 0.00 & 65 & 12.24 & i & 0. & i & 0 & 77 & 14.50 & 0 & 0.00 & 0 & 0.00 & 0 & & 16 & 3.01 & 0 \\
\hline $2 \mathrm{H}-7,10-12$ & 17.87 & 0 & 0.00 & 113 & 21.24 & 0 & 0.00 & 13 & 2.44 & 0 & 0.00 & 5 & 0 & 72 & 13.53 & 0 & 0.00 & 0 & 0.00 & 0 & & 12 & 2.26 & 0 \\
\hline & 18. & 0 & 0. & 167 & 31.87 & 0 & & 75 & 14. & 0 & 0.00 & 1 & 0.19 & 35 & 6.68 & 0 & 0.00 & 0 & 0.00 & 0 & 0.00 & 16 & 3.05 & 0 \\
\hline $2 \mathrm{H}-7,52-54$ & 18.29 & 0 & 0.00 & 48 & 8.91 & 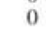 & 00 & 23 & 4.27 & 0 & 0.00 & 2 & 0.37 & 40 & 7.42 & 0 & 0.00 & 0 & 0.00 & 0 & 0.00 & 8 & 1.48 & \\
\hline
\end{tabular}

Figure 1. The Brunhes/Matuyama boundary falls above the base of Gephyrocapsa sp. 3, within the P. lacunosa Zone. The substantial vertically oriented overprint acquired during drilling makes recognition of the normal subchrons within the Matuyama difficult, and both the Jaramillo and the Olduvai subchrons were tentatively recognized on the basis of declination changes (for more details, see Rack et al., this volume). The base of Gephyrocapsa sp. 3 correlates with the top of the Jaramillo, and the base of G. oceanica s.l. occurs at the top of the Olduvai.

\section{Site 871 (Limalok Guyot)}

Quaternary calcareous nannofloras at Site 871 are very similar to those observed at Site 880 (Table 3 and Fig. 2). In the lower Pleistocene, assemblages consist of abundant small and medium-sized Reticulofenestra, Pseudoemiliania lacunosa, and small Gephyrocapsa. Through the lower and middle Pleistocene, although reticulofenestrids and $P$. lacunosa decrease in abundance, the gephyrocapsid group becomes gradually dominant. Also at this site, in the uppermost Pleistocene, Emiliania huxleyi outnumbers all the other nannofossil taxa.

In the middle Pleistocene, the Gephyrocapsa group shows a relative decrease in abundance that is paralleled by a relative increase of P. lacunosa (12.25-8.3 mbsf) and of Reticulofenestra, which is represented exclusively by a large variety (11.8-8.85 mbsf) (Table 3 and Fig. 2).

Within the Gephyrocapsa group, the small Gephyrocapsa and $G$. caribbeanica dominate in the lowermost Pleistocene, whereas $G$. oceanica s.1. appears and gradually increases in abundance upward. The large Gephyrocapsa were observed only between 13.35 and $12.25 \mathrm{mbsf}$, and their top coincides with the base of dominant small Gephyrocapsa. As observed at Site 880, the medium-sized gephyrocapsids are virtually absent in the interval immediately underlying the base of the small Gephyrocapsa Zone. G. oceanica s.I. reenters the section at 9.25 mbsf, where Gephyrocapsa sp. 3 was first observed. The latter taxon is present up to $6.80 \mathrm{mbsf}$.

The first occurrence of Emiliania huxleyi (2.9 mbsf) coincides with a marked change in the gephyrocapsid composition (Fig. 2). In fact, at this level, $G$. caribbeanica sharply decreases in abundance, whereas the small Gephyrocapsa become dominant. In the upper $1.70 \mathrm{~m}$ of the section, a few specimens of large Gephyrocapsa and Gephyrocapsa sp. 3 were observed. The topmost sample (Sample 144-871A-1H-1, 20-
$21 \mathrm{~cm}$ ) also contains very rare specimens of $P$. lacunosa that indicate reworking of older material. However, no evidence of reworking was noticed between 0.82 and $1.70 \mathrm{mbsf}$, and therefore the occurrence of large Gephyrocapsa and Gephyrocapsa sp. 3 might indicate a reentrance of these morphotypes in the latest Pleistocene.

Eleven biostratigraphic events and eight biozones were recognized at this site (Table 6); the depths of the nannofossil events are reported in Table 7.

\section{Site 872 (Lo-En Guyot)}

Although the Quaternary section at Site 872 is much shorter than at Sites 880 and 871,11 nannofossil events and 8 biozones were detected (Table 6 and Fig. 3). The complete sequence of biostratigraphic events suggests that the Pleistocene is complete but characterized by a low sedimentation rate and/or by minor hiatuses that biostratigraphy cannot distinguish. As discussed in Premoli Silva, Haggerty, Rack, et al. (1993), these possible hiatuses may be related to the strong currents that remove even large quantities of unconsolidated sediments from the top of elevated features such as guyots.

Calcareous nannofloras at this site are very similar to those observed at Sites 880 and 871 . In fact, small and medium Reticulofenestra and Pseudoemiliania lacunosa decrease in abundance from the lower to the middle Pleistocene, whereas the gephyrocapsid group becomes gradually dominant. Emiliania huxleyi also becomes very abundant at this site in the uppermost Pleistocene. In the lowermost Pleistocene, between 7.30 and 8.35 mbsf, Florisphaera profunda is common.

As previously reported for Sites 880 and 871 , the Gephyrocapsa group shows a relative decrease in abundance in the middle Pleistocene, where both $P$. lacunosa $(6.25-4.25 \mathrm{mbsf})$ and large Reticulofenestra (5.85-3.25 mbsf) become more abundant (Table 4 and Fig. 3 ).

Distribution patterns similar to those at Sites 880 and 871 were also detected within the Gephyrocapsa group (Fig. 3). In the lowermost Pleistocene, the small Gephyrocapsa dominate, G. caribbeanica is frequent, and $G$. oceanica s.l. is rare. The large Gephyrocapsa were observed in a short interval (7.30-6.25 mbsf) below the small Gephyrocapsa Zone where the small specimens are dominant. G. caribbeanica gradually increases in abundance in the upper part. The top of the small Gephyrocapsa Zone is marked by the reentrance of $G$. oceanica s.l. (4.95 mbsf), which coincides with the appearance of 
Table 2 (continued).

\begin{tabular}{|c|c|c|c|c|c|c|c|c|c|c|c|c|c|c|c|c|c|c|c|c|c|c|c|}
\hline 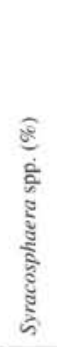 & $\frac{\tilde{\xi}}{\xi}$ & $\begin{array}{c}\hat{d} \\
\hat{y} \\
\hat{v}\end{array}$ & 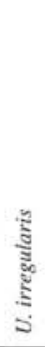 & 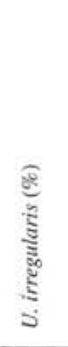 & 昙 & 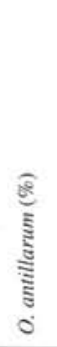 & 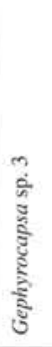 & 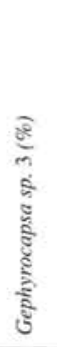 & $\frac{\Xi}{\mathbb{\Xi}}$ & 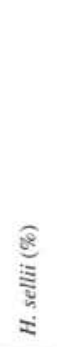 & 胥 & 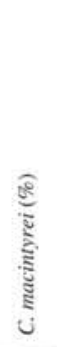 & 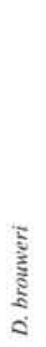 & 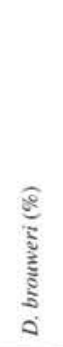 & 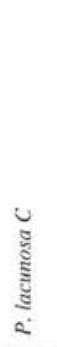 & 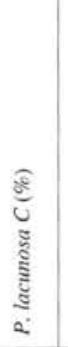 & 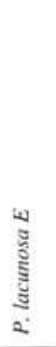 & 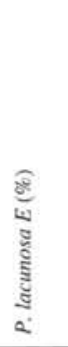 & 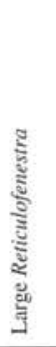 & 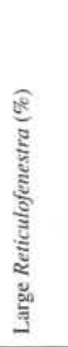 & 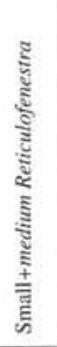 & 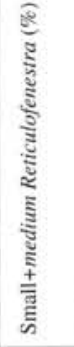 & 푱 \\
\hline 0.00 & 0 & 0.00 & 0 & 0.00 & 0 & 0.00 & 0 & 0.00 & 6 & 1.19 & 6 & 1.19 & 0 & 0.00 & 10 & 1.98 & 115 & 22.73 & 0 & 0.00 & 0 & 0.00 & 506 \\
\hline 0.00 & 0 & 0.00 & 0 & 0.00 & 0 & 0.00 & 0 & 0.00 & 2 & 0.38 & 8 & 1.53 & 0 & 0.00 & 18 & 3.45 & 115 & 22.03 & 0 & 0.00 & 168 & 32.18 & 522 \\
\hline 0.00 & 0 & 0.00 & 0 & 0.00 & 0 & 0.00 & 0 & 0.00 & 0 & 0.00 & 13 & 2.52 & 0 & 0.00 & 10 & 1.94 & 108 & 20.93 & 0 & 0.00 & 123 & 23.84 & 516 \\
\hline 0.19 & 0 & 0.00 & 0 & 0.00 & 0 & 0.00 & 0 & 0.00 & 2 & 0.38 & 15 & 2.87 & 0 & 0.00 & 16 & 3.07 & 82 & 15.71 & 0 & 0.00 & 143 & 27.39 & 522 \\
\hline 0.18 & 0 & 0.00 & 0 & 0.00 & 0 & 0.00 & 0 & 0.00 & 8 & 1.43 & 5 & 0.89 & 0 & 0.00 & 15 & 2.68 & 67 & 11.99 & 0 & 0.00 & 80 & 14.31 & 559 \\
\hline 0.00 & 0 & 0.00 & 0 & 0.00 & 0 & 0.00 & 0 & 0.00 & 1 & 0.19 & 8 & 1.55 & 2 & 0.39 & 3 & 0.58 & 25 & 4.84 & 0 & 0.00 & 62 & 11.99 & 517 \\
\hline 0.00 & 0 & 0.00 & 0 & 0.00 & 0 & 0.00 & 0 & 0.00 & 2 & 0.39 & 10 & 1.95 & 2 & 0.39 & 11 & 2.14 & 109 & 21.21 & 0 & 0.00 & 133 & 25.88 & 514 \\
\hline 0.00 & 0 & 0.00 & 0 & 0.00 & 0 & 0.00 & 0 & 0.00 & 5 & 0.95 & 2 & 0.38 & $i$ & 0.19 & 20 & 3.82 & 122 & 23.28 & 0 & 0.00 & 155 & 29.58 & 524 \\
\hline 0.56 & 0 & 0.00 & 0 & 0.00 & 0 & 0.00 & 0 & & 3 & 0.56 & 8 & 1.5 & 1 & 0.19 & 2 & $\begin{array}{l}3.02 \\
4.14\end{array}$ & 177 & 33,33 & 0 & 0.00 & 167 & 31.45 & 531 \\
\hline 0.19 & 0 & 0.00 & 0 & 0.00 & 0 & 0.00 & 0 & 0.00 & 4 & 0.76 & 7 & 1.3 & 1 & 0.19 & 0 & $\begin{array}{l}4.74 \\
1.90\end{array}$ & 48 & 9.14 & 0 & 0.00 & 82 & 15.62 & 525 \\
\hline 0.00 & 0 & 0.00 & 0 & 0.00 & 0 & 0.00 & 0 & 0.00 & 2 & 0.38 & 8 & 1.50 & 7 & 1.31 & 5 & $\begin{array}{l}2.80 \\
\end{array}$ & 10 & 20.26 & 0 & 0.00 & 122 & 22.89 & 533 \\
\hline 0.19 & 0 & 0.00 & 0 & 0.00 & 0 & 0.00 & 0 & 0.00 & $i$ & 0.19 & 8 & 1.54 & 3 & 0.58 & 12 & 2.31 & 149 & 28.65 & 0 & 0.00 & 165 & 31.73 & 520 \\
\hline 0.00 & 0 & 0.00 & 0 & 0.00 & 0 & 0.00 & 0 & 0.00 & 11 & $\begin{array}{l}0.19 \\
1.99\end{array}$ & 29 & $\begin{array}{l}1.34 \\
5.23\end{array}$ & 8 & $\begin{array}{l}0.58 \\
1.44\end{array}$ & 3 & 0.54 & 117 & $\begin{array}{l}20.03 \\
21.12\end{array}$ & 0 & 0.00 & 178 & 32.13 & 554 \\
\hline 0.00 & 0 & 0.00 & 0 & 0.00 & 0 & 0.00 & 0 & 0.00 & 2 & 0.38 & 8 & 1.5 & 5 & 0. & 3 & 0.56 & 58 & 10.92 & 0 & 0.00 & 222 & 41.81 & 531 \\
\hline 0. & 0 & 0.00 & 0 & 0.00 & 0 & 0.00 & 0 & 0.00 & 2 & 0 . & 15 & 2.8 & 5 & 0 . & 8 & & 180 & 33.83 & 0 & 0.00 & 107 & 20.11 & 532 \\
\hline & 0 & 0 & 0 & 0. & 0 & 0. & 0 & 0.00 & i & & 3 & 0 . & 2 & & 7 & 1.34 & 97 & 18.51 & 0 & 0.00 & 120 & 22.90 & 524 \\
\hline 0.00 & 0 & 0.00 & 0 & 0.00 & 0 & 0.00 & 0 & 0.00 & i & 0.19 & 13 & 2. & II & 1.86 & 47 & 8.72 & 134 & 24.86 & 0 & 0.00 & 213 & 39.52 & 539 \\
\hline
\end{tabular}

Gephyrocapsa sp. 3 . The latter taxon was observed up to $3.85 \mathrm{mbsf}$. Tiny specimens of Gephyrocapsa (2-2.5 $\mu \mathrm{m}$ maximum length) become dominant between 6.85 and $8.35 \mathrm{mbsf}$ at the base of the small Gephyrocapsa Zone.

G. caribbeanica sharply decreases in abundance at $1.35 \mathrm{mbsf}$, where Emiliania huxleyi was first observed. From this level upward, the small specimens dominate the Gephyrocapsa group and $G$. oceanica s.l. is common. As previously reported for Site 872, a few specimens of large Gephyrocapsa and Gephyrocapsa sp. 3 were observed in the uppermost Pleistocene.

\section{Site 873 (Wodejebato Guyot)}

At Site 873, the Quaternary consists of a thin section of foraminifernannofossil ooze $(4.97 \mathrm{~m})$. Calcareous nannofossil biostratigraphy (Fig. 4) indicates that only the lower Pleistocene is represented. The abundance of single nannofossil taxa is reported in Table 5, and the composition of the Gephyrocapsa group is illustrated in Figure 4.

The lower portion of the Quaternary contains abundant small and medium-sized Reticulofenestra, Pseudoemiliania lacunosa, and small Gephyrocapsa. Both G. caribbeanica and G. oceanica s.l. are represented by frequent specimens. From $3.07 \mathrm{mbsf}$ upward, the large Gephyrocapsa observed indicate that the top of the pelagic cap at Site 873 is older than the small Gephyrocapsa Zone. Helicosphaera sellii was observed throughout the sequence, and therefore its top was not detected at this site (Table 5 and Fig. 4). As noted at Site 872, Florisphaera profunda is common in the lower Pleistocene (1.244.47 mbsf).

\section{PLEISTOCENE CALCAREOUS NANNOFOSSIL BIOSTRATIGRAPHY}

The quantitative analysis of the calcareous nannofossils at Sites $880,871,872$, and 873 resulted in a high-resolution biostratigraphy for the Quaternary. Eleven events and eight biozones were identified (Figs, 1-4). Figure 6 illustrates the correlation of the sites studied based on nannofossil biostratigraphy.

The same sequence of nannofossil events was reproduced at Sites 880,871 , and 872 , although the spacing between biohorizons is extremely variable. This reflects the intense current activity on top of guyots, resulting in winnowed nannofossil foraminifer oozes with variable accumulation rates and minor hiatuses that cannot be precisely estimated with biostratigraphy. At Site 873, most of the Quaternary section is missing; only the lower Pleistocene nannofossil events were determined, thus suggesting that the accumulation of recent sediments on Wodejebato Guyot is strongly affected by current activity.

The ages estimated by various authors for nannofossil marker events at Sites $871,872,873$, and 880 are given in Table 6 , and the depths for these events are given in Table 7. As previously concluded by Matsuoka and Okada $(1989,1990)$, most of the Pleistocene nannofossil events are reliable and reproducible in different oceans; therefore, they can be used for global correlations. It must be noted that the top of Helicosphaera sellii is the less precise event in this study because of its very low abundance and apparent diachrony of the last occurrence. Similar patterns were also observed by Raffi et al. (1993) and Wei (1993) and suggest that this event is not reliable for biostratigraphic correlations.

\section{COMPARISON WITH PREVIOUS RESULTS FROM THE PACIFIC OCEAN AND OTHER BASINS}

Quaternary nannofossil assemblages are very similar at the four sites investigated in the present study, suggesting that nannofloras are not affected by latitudinal gradients between $5^{\circ}$ and $34^{\circ} \mathrm{N}$. The influence of local oceanographic conditions on assemblage composition is possibly reflected in temporarily restricted increases in the abundance of particular taxa, such as very small Gephyrocaps $a$ at Sites 880 and 872 and of Florisphaera profunda at Sites 872 and 873. To understand the distribution of Pleistocene calcareous nannofossils and their sensitivity to different water masses better, the data collected during this study were compared with the nannofloral record at other sites in the Pacific Ocean and in other oceanic basins.

Quaternary deposits from the high-productivity area of the central equatorial Pacific were investigated by Pujos (1985a) for their nannofossil content. This semiquantitative study was mainly biostratigraphically oriented, and it is difficult to use the data for ecologic interpretations. A direct comparison is also hampered by the different taxonomic concepts used by Pujos with respect to those adopted in the present study. At Sites 571 and 575, Pujos (1985a) documented the dissolution and reworking of Tertiary taxa at various levels in the Quaternary sections. These events are explained by the episodic influence of corrosive deep-water masses. 


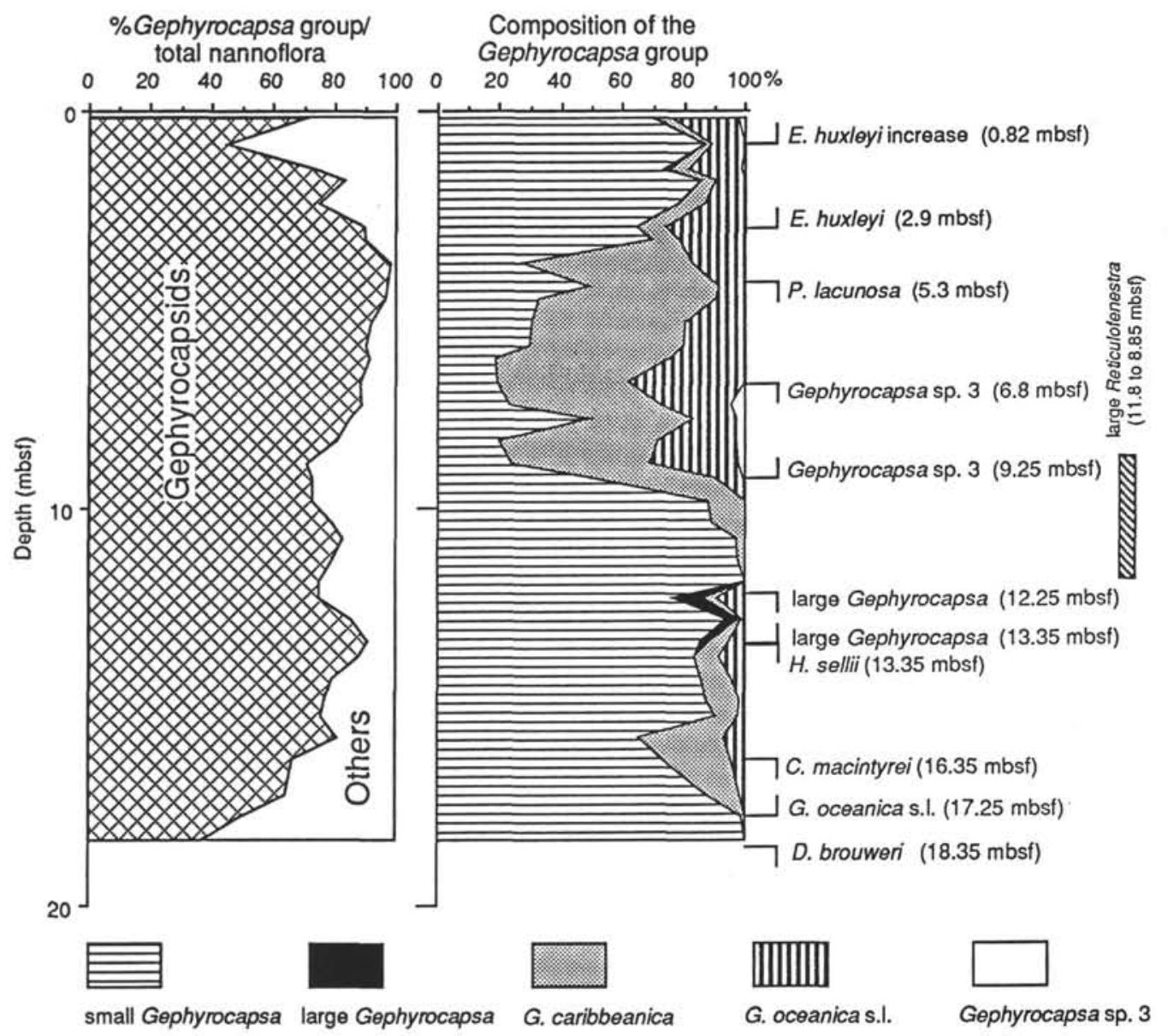

Figure 2. Abundance of gephyrocapsids vs. other nannofossils and composition (percentages) of various taxonomic classes within the Gephyrocapsa group at Site 871 (Limalok Guyot). On the right are the biostratigraphic results of quantitative analyses of nannofloras.

Matsuoka and Okada (1989) described Pleistocene assemblages from a core from the subtropical Pacific, at approximately $20^{\circ} \mathrm{N}$ latitude. Although a direct comparison is difficult because different taxonomic concepts were applied to the Gephyrocapsa group, there are strong similarities between the assemblages documented by Matsuoka and Okada (1989) and those described here. In particular, they reported a gradual decrease in the abundance of Pseudoemiliania lacunosa through the lower Pleistocene, which is interrupted by a distinctive increase in the middle Pleistocene. Small- and mediumsized reticulofenestrids gradually decrease in abundance upward and the large variety (Reticulofenestra A large) was reported only in a short interval within the middle Pleistocene.

At the appearance level of Emiliania huxleyi, Matsuoka and Okada (1989) illustrated a marked decrease in the abundance of the Gephyrocapsa group, which is most probably equivalent to the $G$. caribbeanica decline reported in the present study. Similar to the record at Sites 880 and 872 , marked increases in the abundance of the smallest gephyrocapsids $(2.5-3 \mu \mathrm{m})$ were noticed at the base of the small Gephyrocapsa Zone and close to the last occurrence of large Reticulofenestra. This similarity at three sites located at approximately $10^{\circ} \mathrm{N}, 20^{\circ} \mathrm{N}$, and $34^{\circ} \mathrm{N}$ suggests that these abundance peaks are probably time-controlled rather than paleoceanographically driven. However, more data are needed to prove or disprove this interpretation and for eventual use of these abundance changes for biostratigraphy.

The distribution of Florisphaera profunda reported by Matsuoka and Okada (1989) does not show significant increases in abundance in the lower Pleistocene as those observed at Sites 872 and 873. At the site studied by Matsuoka and Okada (1989), the abundance curve of this species shows limited fluctuations through the Quaternary, with one peak in the upper Pleistocene at the base of the Emiliania huxleyi acme. Increases in abundance of Florisphaera profunda may derive from the selective exclusion of species more sensitive to dissolution or to decreases in production of other coccolithophorids in the upper euphotic zone, as also inferred by Matsuoka and Okada (1989). Sites 872 and 873 are located at water depths of 1084 and 1334 mbsf, respectively. It is unlikely that the increase in abundance of Florisphaera profunda at these sites result from selective dissolution, because the preservation of calcareous nannofloras is generally very good. Further investigations in the Pacific Ocean are necessary to clarify the oceanographic changes that influenced the local blooms of Florisphaera profunda during the Quaternary.

A calibration of nannofossil events with oxygen isotope stratigraphy in the upper Pliocene-lower Pleistocene interval was proposed by Wei (1993). The study was conducted on eight DSDP/ODP sites in the Atlantic, Pacific, and Indian oceans, between $53^{\circ} \mathrm{N}$ and $41^{\circ} \mathrm{S}$ latitudes. Although most events are synchronous, most of the early Pleistocene events seem to be slightly diachronous.

Recently, Raffi et al. (1993) quantified calcareous nannofloras in the lower to middle Pleistocene interval of Sites 677 and 504 and Core V28-239 from the eastern equatorial Pacific and calibrated biostratigraphic events as well as changes in assemblage composition (mainly within the Gephyrocapsa group) with magnetostratigraphy and oxygen isotope stratigraphy. Although Raffi et al. (1993) used a slightly different subdivision of the gephyrocapsids (e.g., the small specimens are smaller than $4 \mu \mathrm{m}$ ), all the events and compositional changes detected in their investigation compare well with those documented 


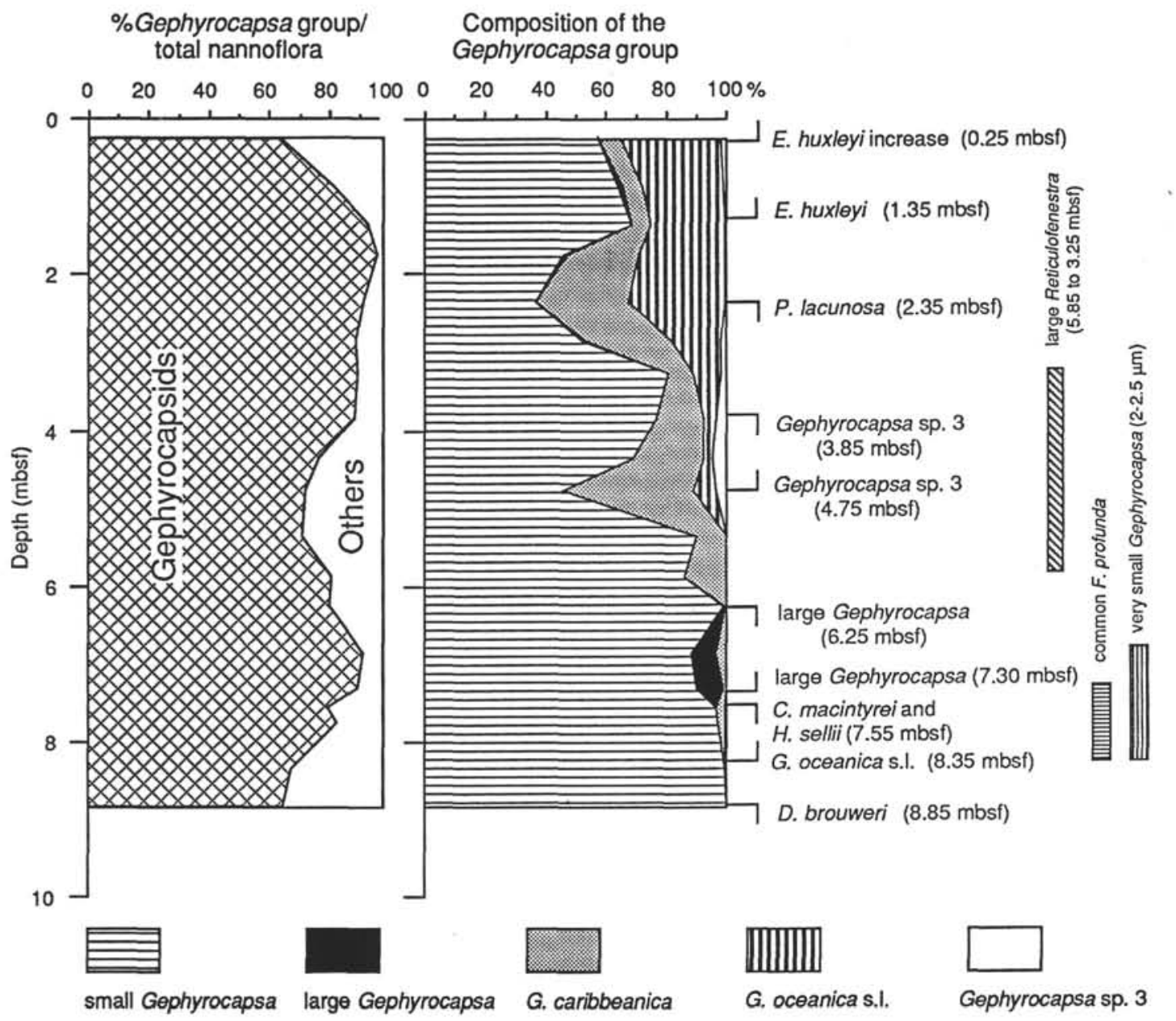

Figure 3. Abundance of gephyrocapsids vs. other nannofossils and composition (percentages) of various taxonomic classes within the Gephyrocapsa group at Site 872 (Lo-En Guyot). On the right are the biostratigraphic results of quantitative analyses of nannofloras.

here. In fact, the changes in abundance of the single Gephyrocapsa classes occur in the same sequence and in equivalent stratigraphic levels. As proposed by Raffi et al. (1993), the base of the small Gephyrocapsa Zone is best characterized by the extinction of the large Gephyrocaps $a$ and the virtual absence of the medium-sized specimens. The top of the small Gephyrocapsa Zone is difficult to identify if the end of the small gephyrocapsid dominance is used. In agreement with Raffi et al. (1993), the present study documented that the top of this zone is better defined by the reentrance of the medium-sized gephyrocapsids, which coincides with the first occurrence of Gephyrocapsa sp. 3. The present study confirms that Gephyrocapsa sp. 3 is present in a short interval in the middle Pleistocene, as reported by Raffi et al. (1993), but few specimens were observed in the uppermost Pleistocene at Sites 871 and 872 and may indicate a reentrance of these morphotypes.

Gartner (1977) first outlined the dominance of small Gephyrocapsa in the middle Pleistocene of the Pacific Ocean and the Caribbean region, and established a biozone - defined as the interval between the last occurrence of Helicosphaera sellii and the end of dominant small Gephyrocapsa - where the small $(<3 \mu \mathrm{m})$ gephyrocapsids dominate the nannofloras. This event was further studied by Gartner (1988), who documented the occurrence of the middle Pleistocene small Gephyrocapsa acme on a global scale and interpreted this episode as the response of calcareous nannoplankton to a global change in the oceanic circulation and productivity.

Comparing the distribution patterns of Quaternary nannofossils that have been compiled from different oceans, the relative proportions of single taxa seem to show similar and coeval changes during the Pleistocene, despite the differences in taxonomic concepts adopted by various specialists. In fact, in addition to the worldwide dominance of small gephyrocapsids that was discussed by Gartner (1988), other changes in abundance as well as morphometric variations of Quaternary nannofloras appear to be reproducible in different basins and at different latitudes (in tropical and equatorial areas). In particular, Matsuoka and Okada (1990) recognized in the tropical Indian Ocean the six nannoflora assemblages that were previously determined in the tropical Pacific Ocean (Matsuoka and Okada, 1989). Their quantitative study of calcareous nannofossils at Site 709 resulted in the identification of time-progressive changes in Quaternary assemblages that are consistent with the record in the Pacific Ocean (Matsuoka and Okada, 1989; Erba, this study). In particular, in the tropical Indian Ocean Reticulofenestra and Pseudoemiliania lacunosa are abundant in the lower Pleistocene and both decline through the middle Pleistocene, whereas gephyrocapsids gradually increase in abundance upward, dominate over all the other nannofossils in the middle to upper Pleistocene, but markedly decrease in abundance in the uppermost Pleistocene, where they are outnumbered mainly by Emiliania huxleyi. In addition, a relative increase in the abundance of Pseudoemiliania lacunosa was reported for the middle Pleistocene.

Calibration of quantitative Pleistocene nannofossil biostratigraphy with oxygen isotope stratigraphy and magnetostratigraphy proved that nannofossil events may be diachronous in different areas (Raffi et al., 1993; Wei, 1993). However, the reproducibility of major changes in 


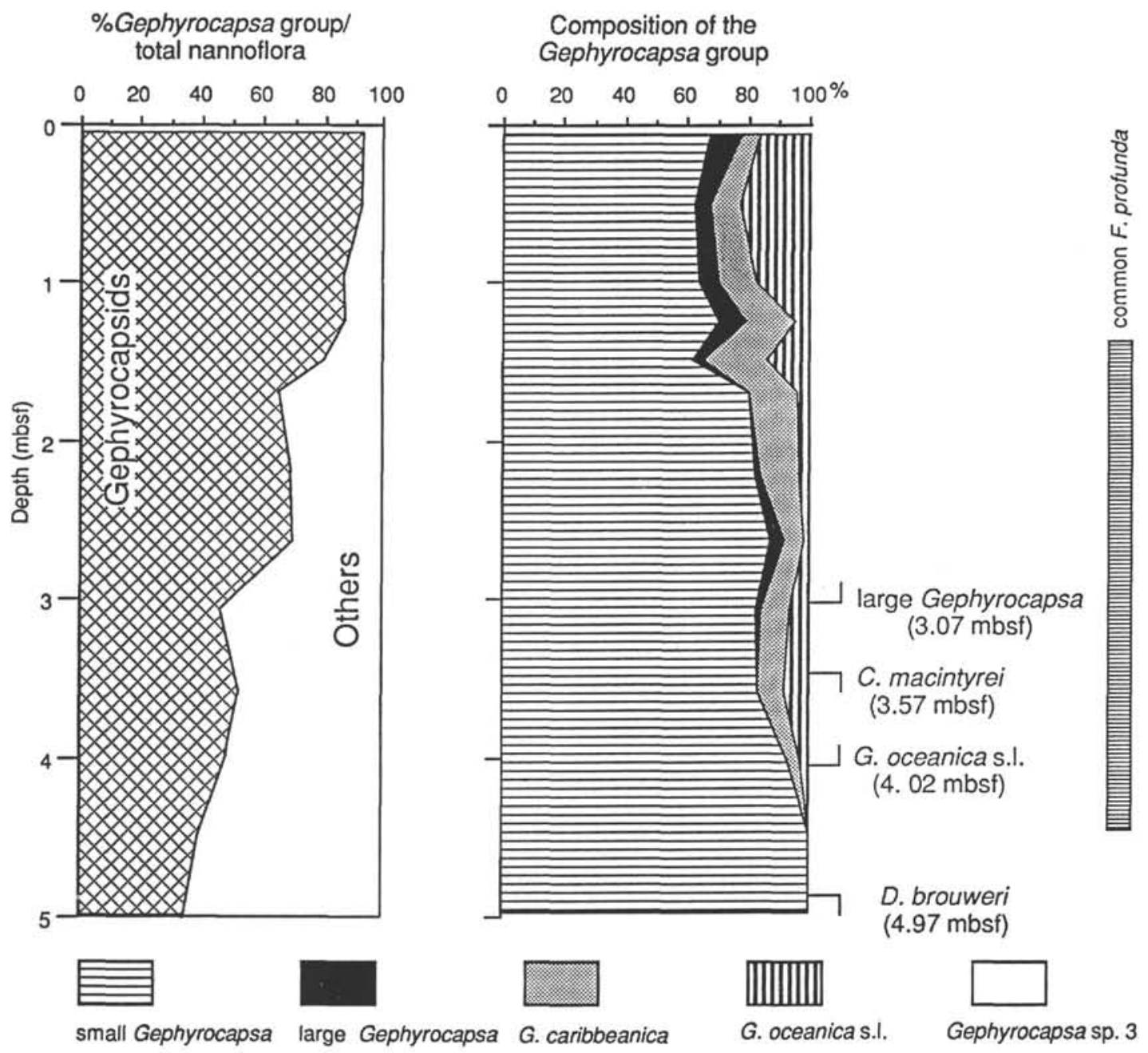

Figure 4. Abundance of gephyrocapsids vs. other nannofossils and composition (percentages) of various taxonomic classes within the Gephyrocapsa group at Site 873 (Wodejebato Guyot). On the right are the biostratigraphic results of quantitative analyses of nannofloras.

distribution patterns of early and middle Pleistocene nannofloras in the Pacific and Atlantic oceans, Caribbean Sea, Mediterranean Sea, and Timor Sea was documented (Raffi et al., 1993).

Middle to upper Pleistocene sediments from the eastern Mediterranean were recently studied for their nannofossil content by Castradori (1993a, 1993b), using quantitative methods and taxonomic concepts consistent with those of Rio et al. (1990), Raffi et al. (1993); these were adopted for the present study. This investigation revealed that calcareous nannofloras in the Mediterranean also show compositional changes comparable with those found on a more global scale, although some local effects were noted. In particular, the distribution of Gephyrocapsa sp. 3 is consistent with that observed in this study and reported by Raffi et al. (1993). The beginning of the "acme" of small Gephyrocapsa close to the first occurrence of Emiliania huxleyi is correlatable with the decline of $G$. caribbeanica and the concomitant increase in abundance of small gephyrocapsids that are documented in this study at the base of the Emiliania huxleyi Zone.

\section{CONCLUSIONS}

Quantitative investigation of calcareous nannofloras at Sites 880, 871,872 , and 873 resulted in a high-resolution biostratigraphy of the Pleistocene and in the characterization of assemblages that display similar changes in composition. The present study suggests that the Pleistocene accumulation of pelagic sediments on guyots in the cen- tral and western Pacific is neither continuous or constant. In fact, a small hiatus was detected at Site 880 (Takuyo-Daisan Guyot) in the upper Pleistocene, whereas most of the middle and the upper Pleistocene is missing on Wodejebato Guyot (Site 873). On Lo-En Guyot (Site 872), a complete sequence of nannofossil events was detected, but the limited thickness of the Quaternary section implies possible minor hiatuses that cannot be identified biostratigraphically. As discussed in Premoli Silva, Haggerty, Rack, et al. (1993), sediment accumulation was strongly controlled by currents winnowing the top of these elevated features.

Distinctive changes in the abundance of nannofossil taxa, especially within the Gephyrocapsa group, were observed at all sites. They seem to be coeval on a regional, and even a global, scale. This suggests that calcareous nannofloral assemblages are not very sensitive to latitudinal gradients, although local oceanographic effects can influence specific taxa, such as the smallest specimens of Gephyrocapsa $(2-2.5 \mu \mathrm{m})$ and Florisphaera profunda.

\section{ACKNOWLEDGMENTS}

I wish to express my gratitude to Isabella Premoli Silva and Janet Haggerty for their enthusiastic leadership during Leg 144. Sincere thanks are extended to Frank Rack and the Leg 144 Scientific Party for discussion and cooperation. A special note of thanks to Jeff Gee for providing paleomagnetic data from Site 880 , as well as for suggestions 

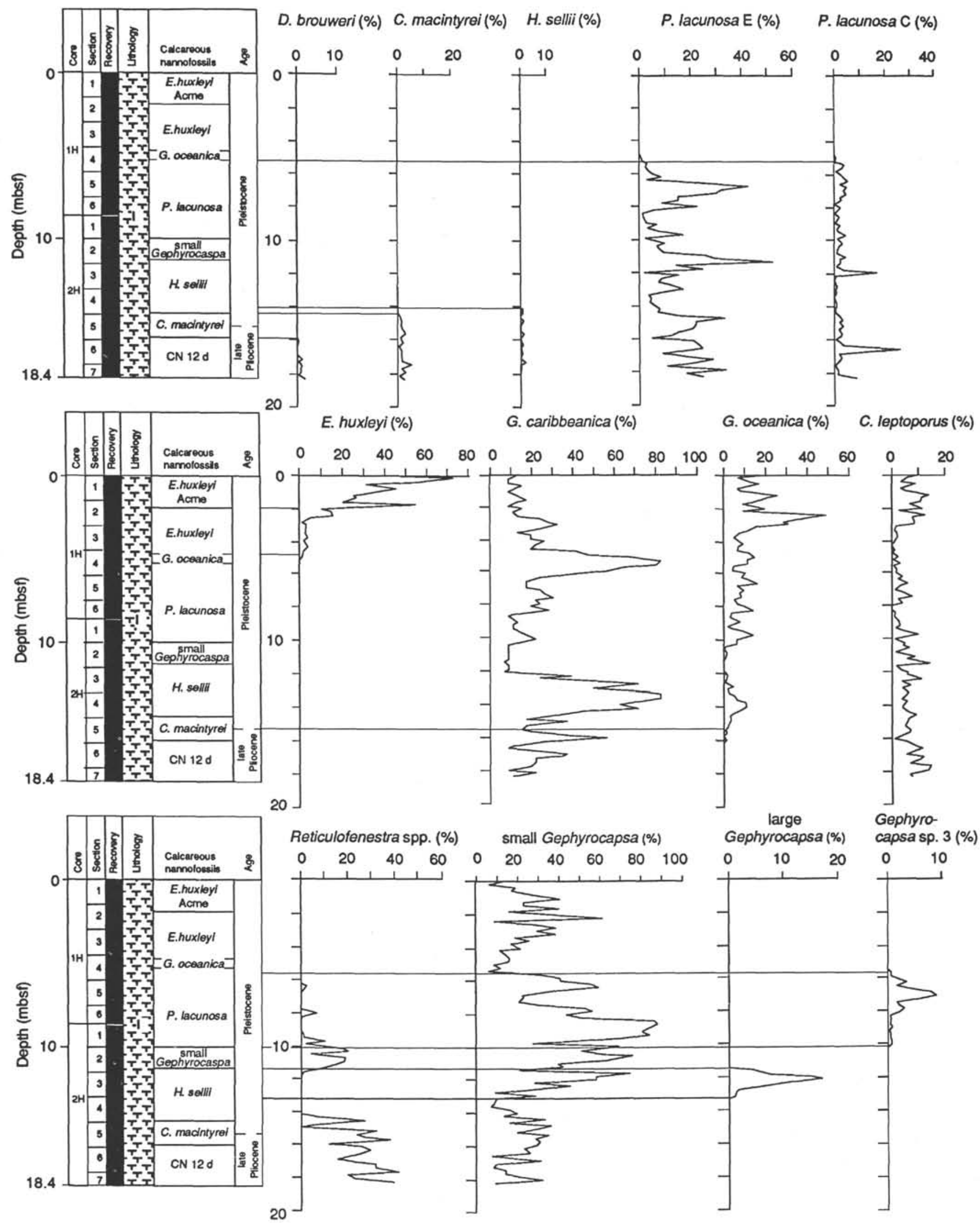

Figure 5. Percentage plots of the biostratigraphically important nannofossil taxa in the Quaternary sediments recovered at Site 880 (Takuyo-Daisan Guyot). Curves of the most abundant taxa are also reported. 
Table 3. Quantitative distribution of Quaternary calcareous nannofossils at Site 871 (Limalok Guyot).

\begin{tabular}{|c|c|c|c|c|c|c|c|c|c|c|c|c|c|c|c|c|c|c|c|c|c|c|c|c|c|}
\hline $\begin{array}{l}\text { Core, section, } \\
\text { interval }(\mathrm{cm})\end{array}$ & $\begin{array}{l}\text { Depth } \\
\text { (mbsf) }\end{array}$ & 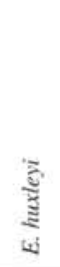 & 氖 & 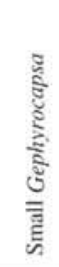 & 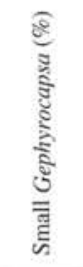 & 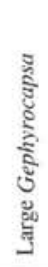 & 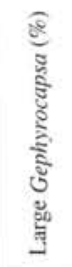 & 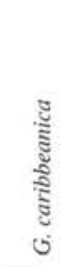 & 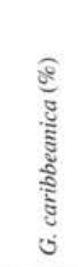 & 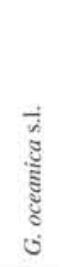 & 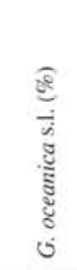 & $\underset{\mathrm{g}}{\mathrm{g}}$ & 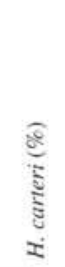 & טั & 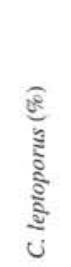 & Uั & 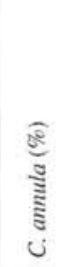 & 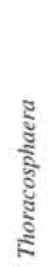 & 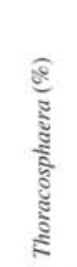 & 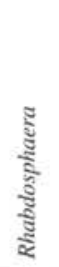 & 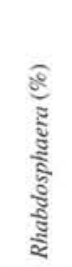 & 芯 & 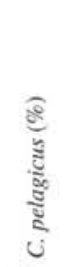 & & 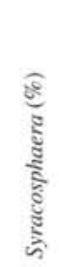 \\
\hline \multicolumn{26}{|l|}{$144-871 \mathrm{~A}=$} \\
\hline $1 \mathrm{H}-1,20-21$ & 0.20 & 107 & 19.81 & 263 & 48.70 & 2 & 0.37 & 23 & 4.26 & 80 & 14.81 & 7 & 1.30 & 16 & 2.96 & 7 & 1.30 & 2 & 0.37 & 2 & 0.37 & 0 & 0.00 & 2 & 0.37 \\
\hline $1 \mathrm{H}-1,82-83$ & 0.82 & 237 & 44.30 & 208 & 38.88 & 0 & 0.00 & 5 & 0.93 & 27 & 5.05 & 2 & 0.37 & 5 & 0.93 & 13 & 2.43 & 2 & 0.37 & 2 & 0.37 & 0 & 0.00 & 3 & 0.56 \\
\hline $1 \mathrm{H}-1,143-144$ & 1.43 & 75 & 14.31 & 283 & 54.01 & 7 & 1.34 & 25 & 4.77 & 67 & 12.79 & 2 & 0.38 & 5 & 0.95 & 20 & 3.82 & 2 & 0.38 & 2 & 0.38 & 0 & 0.00 & 2 & 0.38 \\
\hline $1 \mathrm{H}-2,20-21$ & 1.70 & 40 & 7.87 & 358 & 70.47 & $i$ & 0.20 & 19 & 3.74 & 43 & 8.46 & 3 & 0.59 & 5 & 0.98 & 8 & 1.57 & 2 & 0.39 & 2 & 0.39 & 0 & 0.00 & 3 & 0.59 \\
\hline $1 \mathrm{H}-2,80-81$ & 2.30 & 73 & 14.43 & 295 & 58.30 & 0 & 0.00 & 33 & 6.52 & 52 & 10.28 & 8 & 1.58 & 7 & 1.38 & 5 & 0.99 & 1 & 0.20 & 1 & 0.20 & 0 & 0.00 & 1 & 0.20 \\
\hline $1 \mathrm{H}-2,140-141$ & 2.90 & 11 & 2.19 & 291 & 57.85 & 0 & 0.00 & 41 & 8.15 & 118 & 23.46 & 5 & 0.99 & 2 & 0.40 & 1 & 0.20 & 5 & 0.99 & 0 & 0.00 & 0 & 0.00 & 3 & 0.60 \\
\hline $1 \mathrm{H}-3,20-21$ & 3.20 & 5 & 0.98 & 318 & 62.23 & 0 & 0.00 & 43 & 8.41 & 100 & 19.57 & 3 & 0.59 & 2 & 0.39 & 17 & 3.33 & 2 & 0.39 & 1 & 0.20 & 0 & 0.00 & 5 & 0.98 \\
\hline $1 \mathrm{H}-3,80-81$ & 3.80 & 0 & 0.00 & 135 & 26.73 & 0 & 0.00 & 272 & 53.86 & 88 & 17.43 & 1 & 0.20 & 2 & 0.40 & 3 & 0.59 & 1 & 0.20 & 0 & 0.00 & 0 & 0.00 & 1 & 0.20 \\
\hline $1 \mathrm{H}-3,140-141$ & 4.40 & 0 & 0.00 & 240 & 47.06 & 0 & 0.00 & 211 & 41.37 & 44 & 8.63 & 2 & 0.39 & 2 & 0.39 & 5 & 0.98 & 0 & 0.00 & 0 & 0.00 & 0 & 0.00 & 2 & 0.39 \\
\hline $1 \mathrm{H}-4,20-21$ & 4.70 & 0 & 0.00 & 157 & 31.27 & 0 & 0.00 & 277 & 55.18 & 50 & 9.96 & 4 & 0.80 & 4 & 0.80 & 7 & 1.39 & i & 0.20 & 0 & 0.00 & 0 & 0.00 & 0 & 0.00 \\
\hline $1 \mathrm{H}-4,80-81$ & 5.30 & 0 & 0.00 & 143 & 27.77 & 0 & 0.00 & 233 & 45.24 & 95 & 18.45 & $i$ & 0.19 & 10 & 1.94 & 6 & 1.17 & 0 & 0.00 & 0 & 0.00 & 0 & 0.00 & 6 & 1.17 \\
\hline $1 \mathrm{H}-4,140-141$ & 5.90 & 0 & 0.00 & 135 & 26.68 & 0 & 0.00 & 225 & 44.47 & 94 & 18.58 & 6 & 1.19 & 8 & 1.5 & 3 & 0.59 & I & 0.20 & 0 & 0.00 & 0 & 0.00 & 0 & 0.00 \\
\hline $1 \mathrm{H}-5,20-21$ & 6.20 & 0 & 0.00 & 88 & 17.15 & 0 & 0.00 & 261 & 50.88 & 118 & 23.00 & 7 & 1.36 & 8 & 1.56 & 0 & 0.00 & I & 0.19 & 0 & 0.00 & 0 & 0.00 & 2 & 0.39 \\
\hline $1 \mathrm{H}-5,80-81$ & 6.80 & 0 & 0.00 & 85 & 16.70 & 0 & 0.00 & 193 & 37.92 & 170 & 33.40 & 2 & 0.39 & 2 & 0.39 & 3 & 0.59 & 0 & 0.00 & 0 & 0.00 & 0 & 0.00 & 0 & 0.00 \\
\hline $1 \mathrm{H}-\mathrm{CC}, 20-21$ & 7.38 & 0 & 0.00 & 110 & 20.91 & 0 & 0.00 & 228 & 43.35 & 108 & 20.53 & 4 & 0.76 & 4 & 0.76 & 5 & 0.95 & 0 & 0.00 & 0 & 0.00 & 0 & 0.00 & 3 & 0.57 \\
\hline $2 \mathrm{H}-1,25-26$ & 7.75 & 0 & 0.00 & 218 & 41.29 & 0 & 0.00 & 151 & 28.60 & 63 & 11.93 & 7 & 1.33 & 14 & 2.65 & 0 & 0.00 & i & 0.19 & 0 & 0.00 & 0 & 0.00 & 4 & 0.76 \\
\hline $2 \mathrm{H}-1,80-81$ & 8.30 & 0 & 0.00 & 82 & 16.02 & 0 & 0.00 & 212 & 41.41 & 105 & 20.51 & 15 & 2.93 & 8 & 1.56 & 5 & 0.98 & $i$ & 0.20 & 0 & 0.00 & 0 & 0.00 & 0 & 0.00 \\
\hline $2 \mathrm{H}-1,135-136$ & 8.85 & 0 & 0.00 & $\begin{array}{l}02 \\
90\end{array}$ & 16.89 & 0 & 0.00 & 168 & 31.52 & 110 & 20.64 & 4 & 0.75 & 8 & 1.5 & 2 & 0.38 & 0 & 0.00 & 0 & 0.00 & 0 & 0.00 & 0 & 0.00 \\
\hline $2 \mathrm{H}-2,25-26$ & 9.25 & 0 & 0.00 & 185 & 36.06 & 0 & 0.00 & 150 & 29.24 & 35 & 6.82 & 7 & 1.36 & 5 & 0.97 & 3 & 0.5 & 1 & 0.19 & 0 & 0.00 & 0 & 0.00 & 2 & 0.39 \\
\hline $2 \mathrm{H}-2,80-81$ & 9.80 & 0 & 0.00 & 331 & 63.17 & 0 & 0.00 & 48 & 9.16 & 0 & 0.00 & 3 & 0.57 & 15 & 2.86 & 5 & 0.95 & 0 & 0.00 & 0 & 0.00 & 0 & 0.00 & 2 & 0.38 \\
\hline $2 \mathrm{H}-2,135-136$ & 10.35 & 0 & 0.00 & 373 & 70.11 & 0 & 0.00 & 46 & 8.65 & 2 & 0.38 & 7 & 1.32 & 8 & 1.50 & 5 & 0.94 & 0 & 0.00 & 0 & 0.00 & 0 & 0.00 & 1 & 0.19 \\
\hline $2 \mathrm{H}-3,25-26$ & 10.75 & 0 & 0.00 & 408 & 80.00 & 0 & 0.00 & 12 & 2.35 & 0 & 0.00 & 5 & 0.98 & 4 & 0.78 & 2 & 0.39 & 0 & 0.00 & 0 & 0.00 & 0 & 0.00 & 0 & 0.00 \\
\hline $2 \mathrm{H}-3,80-81$ & 11.30 & 0 & 0.00 & 386 & 76.89 & 0 & 0.00 & 8 & 1.59 & i & 0.20 & 8 & 1.59 & 4 & 0.80 & 4 & 0.80 & 1 & 0.20 & 0 & 0.00 & 0 & 0.00 & 0 & 0.00 \\
\hline $2 \mathrm{H}-3,135-136$ & 11.85 & 0 & 0.00 & 386 & 74.66 & 0 & 0.00 & 0 & 0.00 & 0 & 0.00 & 2 & 0.39 & 4 & 0.77 & 2 & 0.39 & 0 & 0.00 & 0 & 0.00 & 0 & 0.00 & 1 & 0.19 \\
\hline $2 \mathrm{H}-4,25-26$ & 12.25 & 0 & 0.00 & 305 & 56.80 & 40 & 7.45 & 20 & 3.72 & 37 & 6.89 & 2 & 0.37 & 14 & 2.61 & 2 & 0.37 & 0 & 0.00 & 0 & 0.00 & 0 & 0.00 & 1 & 0.19 \\
\hline $2 \mathrm{H}-4,80-81$ & 12.80 & 0 & 0.00 & 403 & 79.64 & 17 & 3.36 & 5 & 0.99 & 7 & 1.38 & 2 & 0.40 & 15 & 2.96 & 2 & 0.40 & 0 & 0.00 & 0 & 0.00 & 0 & 0.00 & 0 & 0.00 \\
\hline $2 \mathrm{H}-4,135-136$ & 13.35 & 0 & 0.00 & 388 & 77.29 & 12 & 2.39 & 30 & 5.98 & 24 & 4.78 & 15 & 2.99 & i1 & 2.19 & 2 & 0.40 & 1 & 0.20 & 0 & 0.00 & 0 & 0.00 & 0 & 0.00 \\
\hline $2 \mathrm{H}-5,25-26$ & 13.75 & 0 & 0.00 & 382 & 72.49 & 0 & 0.00 & 37 & 7.02 & 42 & 7.97 & 27 & 5.12 & 16 & 3.04 & 2 & 0.38 & 0 & 0.00 & 0 & 0.00 & 0 & 0.00 & 0 & 0.00 \\
\hline $2 \mathrm{H}-5,80-81$ & 14.30 & 0 & 0.00 & 365 & 67.10 & 0 & 0.00 & 42 & 7.72 & 21 & 3.86 & 7 & 1.29 & 16 & 2.94 & 20 & 3.68 & 1 & 0.18 & 0 & 0.00 & 0 & 0.00 & 3 & 0.55 \\
\hline $2 \mathrm{H}-5,135-136$ & 14.85 & 0 & 0.00 & 360 & 66.91 & 0 & 0.00 & 45 & 8.36 & 8 & 1.49 & 5 & 0.93 & 7 & 1.30 & 12 & 2.23 & 0 & 0.00 & 1 & 0.19 & 0 & 0.00 & 3 & 0.56 \\
\hline $2 \mathrm{H}-6,25-26$ & 15.25 & 0 & 0.00 & 365 & 68.10 & 0 & 0.00 & 30 & 5.60 & 10 & 1.87 & 10 & 1.87 & 13 & 2.43 & 8 & 1.49 & 0 & 0.00 & 0 & 0.00 & 0 & 0.00 & 4 & 0.75 \\
\hline $2 \mathrm{H}-6,80-8 \mathrm{I}$ & 15.80 & 0 & 0.00 & 292 & 52.42 & 0 & 0.00 & 127 & 22.80 & 31 & $\begin{array}{l}5.57 \\
5.57\end{array}$ & 14 & 2.51 & 20 & 3.59 & $\frac{0}{7}$ & 1.26 & 0 & 0.00 & 0 & 0.00 & 0 & 0.00 & 4 & 0.72 \\
\hline $2 \mathrm{H}-6,135-136$ & 16.35 & 0 & 0.00 & 283 & 47.88 & 0 & 0.00 & 90 & 15.23 & 18 & 3.05 & 11 & 1.86 & 25 & 4.2. & 48 & 8.12 & 1 & 0.17 & 0 & 0.00 & 0 & 0.00 & 3 & 0.51 \\
\hline $3 \mathrm{H}-1,25-26$ & 17.25 & 0 & 0.00 & 295 & 55,66 & 0 & 0.00 & 37 & 6.98 & 7 & 1.32 & 17 & 3.21 & 15 & 2.83 & 1 & 0.19 & i & 0.19 & 0 & 0.00 & 2 & 0.38 & i & 0.19 \\
\hline $3 \mathrm{H}-1,80-81$ & 17.80 & 0 & 0.00 & 275 & 48.67 & 0 & 0.00 & 3 & 0.53 & 0 & 0.00 & 15 & 2.65 & 42 & 7.43 & 15 & 2.65 & 0 & 0.00 & 0 & 0.00 & 5 & 0.88 & 0 & 0.00 \\
\hline $3 \mathrm{H}-11,135-136$ & 18.35 & 0 & 0.00 & 195 & 36.79 & 0 & 0.00 & 0 & 0.00 & 0 & 0.00 & 7 & 1.32 & 9 & 1.70 & 5 & 0.94 & 1 & 0.19 & 0 & 0.00 & 0 & 0.00 & 2 & 0.38 \\
\hline
\end{tabular}


Table 3 (continued).

\begin{tabular}{|c|c|c|c|c|c|c|c|c|c|c|c|c|c|c|c|c|c|c|c|c|c|}
\hline $\begin{array}{l}\text { Core, section, } \\
\text { interval }(\mathrm{cm})\end{array}$ & 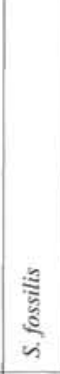 & 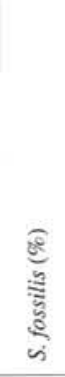 & 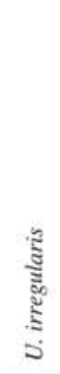 & 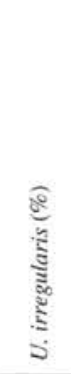 & 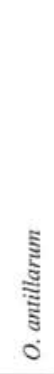 & 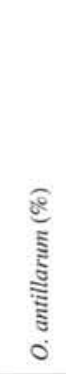 & $\begin{array}{l}\text { m } \\
\text { के } \\
\text { है } \\
\text { हूँ } \\
\text { हैं } \\
\text { हैं }\end{array}$ & 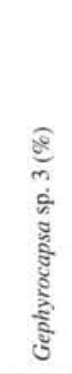 & 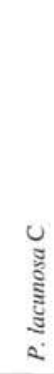 & 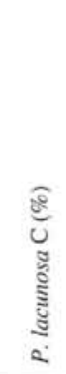 & $\begin{array}{l}\text { wू } \\
\text { हूँ } \\
\text { हूँ } \\
0\end{array}$ & 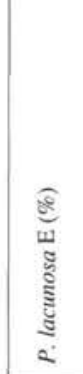 & 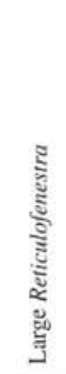 & 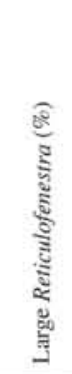 & 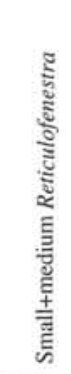 & 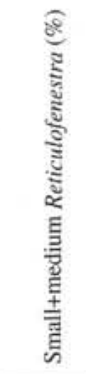 & 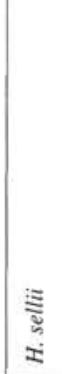 & 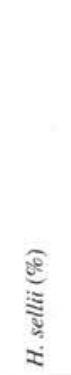 & $\stackrel{\mathrm{\Xi}}{\mathrm{\Xi}}$ & 这 & इ্ \\
\hline 144-871A- & & & & & & & & & & & & & & & & & & & & & \\
\hline $1 \mathrm{H}-1,20-21$ & 5 & 0.93 & 12 & 2.22 & 0 & 0.00 & 10 & 1.85 & 2 & 0.37 & 0 & 0.00 & 0 & 0.00 & 0 & 0.00 & 0 & 0.00 & 0 & 0.00 & 540 \\
\hline $1 \mathrm{H}-1,82-83$ & 8 & 1.50 & 23 & 4.30 & 0 & 0.00 & 0 & 0.00 & 0 & 0.00 & 0 & 0.00 & 0 & 0.00 & 0 & 0.00 & 0 & 0.00 & 0 & 0.00 & 535 \\
\hline $1 \mathrm{H}-1,1$ & 4 & 0.76 & 25 & 4.77 & 0 & 0.0 & 5 & 0. & 0 & 0.0 & 0 & 0.00 & 0 & 0.00 & 0 & 0.00 & 0 & 0.00 & 0 & 0.00 & 524 \\
\hline $1 \mathrm{H}-2,20-21$ & 2 & 0.39 & 20 & 3.94 & 0 & 0.00 & 0 & 0.00 & 0 & 0.0 & 0 & 0.00 & 0 & 0.0 & 0 & 0.0 & 0 & 0.00 & 0 & 0.00 & 508 \\
\hline $1 \mathrm{H}-2,80-81$ & 25 & 4.94 & 2 & 0.40 & 3 & 0.59 & 0 & 0.00 & 0 & 0.00 & 0 & 0.00 & 0 & 0.00 & 0 & 0.00 & 0 & 0.00 & 0 & 0.00 & 506 \\
\hline $1 \mathrm{H}-2,140-141$ & 2 & 0.40 & 24 & 4.77 & 0 & 0.00 & 0 & 0.00 & 0 & 0.00 & 0 & 0.00 & 0 & 0.00 & 0 & 0.00 & 0 & 0.00 & 0 & 0.00 & 503 \\
\hline $1 \mathrm{H}-3,20-2 \mathrm{I}$ & 2 & 0.39 & 13 & 2.54 & 0 & 0.00 & 0 & 0.00 & 0 & 0.00 & 0 & 0.00 & 0 & 0.00 & 0 & 0.00 & 0 & 0.00 & 0 & 0.00 & 511 \\
\hline $1 \mathrm{H}-3,80-81$ & 0 & 0.00 & 2 & 0.40 & 0 & 0.00 & 0 & 0.00 & 0 & 0.00 & 0 & 0.00 & 0 & 0.00 & 0 & 0.00 & 0 & 0.00 & 0 & 0.00 & 505 \\
\hline $1 \mathrm{H}-3,140-141$ & 2 & 0.39 & 2 & 0.39 & 0 & 0.00 & 0 & 0.00 & 0 & 0.00 & 0 & 0.00 & 0 & 0.00 & 0 & 0.00 & 0 & 0.00 & 0 & 0.00 & 510 \\
\hline $1 \mathrm{H}-4,20-21$ & 0 & 0.00 & 2 & 0.40 & 0 & 0.00 & 0 & 0.00 & 0 & 0.00 & 0 & 0.00 & 0 & 0.00 & 0 & 0.00 & 0 & 0.00 & 0 & 0.00 & 502 \\
\hline $1 \mathrm{H}-4,80-81$ & 0 & 0.00 & 4 & 0.78 & 0 & 0.00 & 0 & 0.00 & 4 & 0.78 & 13 & 2.52 & 0 & 0.00 & 0 & 0.00 & 0 & 0.00 & 0 & 0.00 & 515 \\
\hline $1 \mathrm{H}-4,140-141$ & 0 & 0.00 & 1 & 0.20 & 0 & 0.00 & 0 & 0.00 & 13 & 2.57 & 20 & 3.95 & 0 & 0.00 & 0 & 0.00 & 0 & 0.00 & 0 & 0.00 & 506 \\
\hline $1 \mathrm{H}-5,20-2 \mathrm{i}$ & 1 & 0.19 & 5 & 0.97 & 2 & 0.39 & 0 & 0.00 & 8 & 1.56 & 12 & 2.34 & 0 & 0.00 & 0 & 0.00 & 0 & 0.00 & 0 & 0.00 & 513 \\
\hline $\mathrm{IH}-5,80-8 \mathrm{i}$ & 0 & 0.00 & 4 & 0.79 & 0 & 0.00 & 2 & 0.39 & 10 & 1.96 & 38 & 7.47 & 0 & 0.00 & 0 & 0.00 & 0 & 0.00 & 0 & 0.00 & 509 \\
\hline IH-CC, $20-21$ & 9 & 1.71 & 2 & 0.38 & 0 & 0.00 & 22 & 4.18 & 8 & 1.52 & 23 & 4.37 & 0 & 0.00 & 0 & 0.00 & 0 & 0.00 & 0 & 0.00 & 526 \\
\hline $2 \mathrm{H}-1,25-26$ & 5 & 0.95 & 0 & 0.00 & 0 & 0.00 & 15 & 2.84 & 10 & 1.89 & 38 & 7.20 & 0 & 0.00 & 0 & 0.00 & 0 & 0.00 & 0 & 0.00 & 528 \\
\hline $2 \mathrm{H}-1,80-81$ & 0 & 0.00 & 4 & 0.78 & 0 & 0.00 & 13 & 2.54 & 10 & 1.95 & 57 & 11.13 & 0 & 0.00 & 0 & 0.00 & 0 & 0.00 & 0 & 0.00 & 512 \\
\hline $2 \mathrm{H}-1,135-136$ & 5 & 0.94 & 5 & 0.94 & 0 & 0.1 & 10 & 1.88 & 18 & 3.38 & 108 & 20.26 & 5 & 0.9 & 0 & 0.0 & 0 & 0.00 & 0 & 0.00 & 533 \\
\hline $2 \mathrm{H}-2,25-26$ & 1 & 0.19 & 3 & 0.58 & 0 & 0.0 & 2 & 0.39 & 10 & 1.95 & 107 & 20.86 & 2 & 0.3 & 0 & 0.0 & 0 & 0.00 & 0 & 0.00 & 513 \\
\hline $2 \mathrm{H}-2,80-81$ & 0 & 0.00 & 10 & 1.91 & 2 & 0.3 & 0 & 0.00 & 8 & 1.53 & 83 & 15.84 & 17 & 3.2 & 0 & 0.00 & 0 & 0.00 & 0 & 0.00 & 524 \\
\hline $2 \mathrm{H}-2,135-136$ & 3 & 0.56 & 5 & 0.94 & 2 & 0.38 & 0 & 0.00 & 10 & 1.88 & 50 & 9.40 & 20 & 3.7 & 0 & 0.00 & 0 & 0.00 & 0 & 0.00 & 532 \\
\hline $2 \mathrm{H}-3,25-26$ & 0 & 0.00 & 6 & 1.18 & i & 0.20 & 0 & 0.00 & 5 & 0.98 & 57 & 11.18 & 10 & 1.9 & 0 & 0.00 & 0 & 0.00 & 0 & 0.00 & 510 \\
\hline $2 \mathrm{H}-3,80-81$ & 1 & 0.20 & 4 & 0.80 & 0 & 0.00 & 0 & 0.00 & 15 & 2.99 & 68 & 13.55 & 2 & 0.4 & 0 & 0.00 & 0 & 0.00 & 0 & 0.00 & 502 \\
\hline $2 \mathrm{H}-3,135-136$ & 1 & 0.19 & 3 & 0.58 & 1 & 0.19 & 0 & 0.00 & 12 & 2.32 & 90 & 17,41 & 15 & 2.9 & 0 & 0.00 & 0 & 0.00 & 0 & 0.00 & 517 \\
\hline $2 \mathrm{H}-4,25-26$ & 1 & 0.19 & 3 & 0.56 & 0 & 0.0 & 0 & 0.00 & 14 & 2.61 & 98 & 18.25 & 0 & 0.0 & 0 & 0.0 & 0 & 0.00 & 0 & 0.00 & 537 \\
\hline $2 \mathrm{H}-4,80-8$ & 1 & 0.2 & 2 & 0.4 & 0 & 0. & 0 & 0.0 & 5 & 0. & 47 & 9.2 & 0 & 0.0 & 0 & 0.0 & 0 & 0. & 0 & 0.00 & 506 \\
\hline $2 \mathrm{H}-4,135-136$ & 1 & 0.20 & 0 & 0.00 & 0 & 0. & 0 & 0.0 & 3 & 0.6 & 13 & 2. & 0 & 0. & 0 & 0.00 & 1 & 0.20 & 0 & 0.00 & 502 \\
\hline $2 \mathrm{H}-5,25-$ & 0 & 0.00 & 2 & 0.38 & 1 & 0.19 & 0 & 0.00 & 2 & 0.38 & 11 & 2.09 & 0 & 0.0 & 0 & 0.00 & 5 & 0.95 & 0 & 0.00 & 527 \\
\hline $2 \mathrm{H}-5,80-81$ & 0 & 0.00 & 5 & 0.92 & 0 & 0.00 & 0 & 0.00 & 8 & 1.47 & 43 & 7.90 & 0 & 0.0 & 11 & 2.02 & 2 & 0.37 & 0 & 0.00 & 544 \\
\hline $2 \mathrm{H}-5,135-136$ & 0 & 0.00 & 4 & 0.74 & 0 & 0.00 & 0 & 0.00 & 7 & 1.30 & 72 & 13.38 & 0 & 0.0 & 7 & 1.30 & 6 & 1.12 & 0 & 0.00 & 538 \\
\hline $2 \mathrm{H}-6,25-26$ & 0 & 0.0 & 7 & 1.31 & 0 & 0. & 0 & 0.00 & 15 & 2.80 & 63 & 11.75 & 0 & 0.00 & 7 & 1.31 & 4 & 0.75 & 0 & 0.00 & 536 \\
\hline & 0 & 0.0 & 6 & 1.0 & 11 & 1. & 0 & 0.0 & 5 & 0.90 & 23 & 4.1 & 0 & 000 & 12 & 215 & 5 & 0.90 & 0 & 000 & 557 \\
\hline & 0 & 0 . & 5 & 0. & 27 & 4. & 0 & & 8 & & 32 & 5. & 0 & & 26 & & 11 & & 3 & & 1 \\
\hline $3 \mathrm{H}-1,25$ & 0 & 0.00 & 2 & 0.38 & 2 & 0.3 & 0 & 0.00 & 17 & 3. & 92 & 17.36 & 0 & 0. & 15 & 2.83 & 21 & 3. & 5 & 0.94 & 530 \\
\hline $3 \mathrm{H}-1,80-81$ & 0 & 0.00 & 2 & 0.35 & 15 & 2.65 & 0 & 0.00 & 17 & 3.01 & 60 & 10.62 & 0 & 0.00 & 93 & 16.46 & 12 & 2.12 & 11 & 1.95 & 565 \\
\hline $3 \mathrm{H}-11,135-136$ & 0 & 0.00 & 0 & 0.00 & 5 & 0.94 & 0 & 0.00 & 7 & 1.32 & 58 & 10.94 & 0 & 0.00 & 229 & 43.21 & 4 & 0.75 & 8 & 1.51 & 530 \\
\hline
\end{tabular}


Table 4. Quantitative distribution of Quaternary calcareous nannofossils at Site 872 (Lo-En Guyot).

\begin{tabular}{|c|c|c|c|c|c|c|c|c|c|c|c|c|c|c|c|c|c|c|c|c|c|c|c|c|c|c|}
\hline $\begin{array}{l}\text { Core, section, } \\
\text { interval }(\mathrm{cm})\end{array}$ & $\begin{array}{r}\text { Depth } \\
\text { (mbsf) }\end{array}$ & 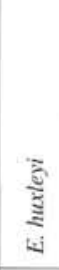 & 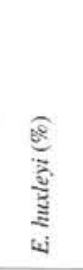 & 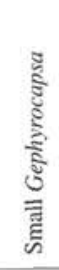 & 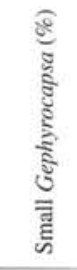 & 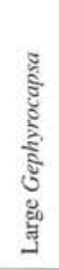 & 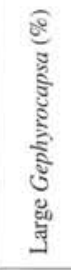 & 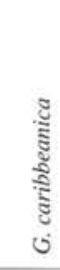 & 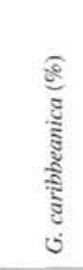 & 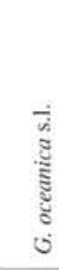 & 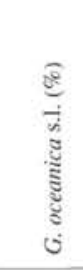 & 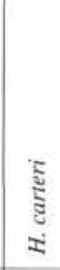 & 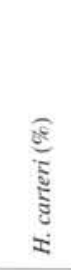 & 气ू & 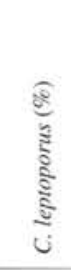 & 竎 & ن & 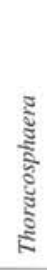 & 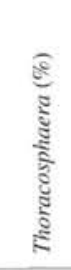 & 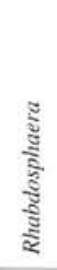 & 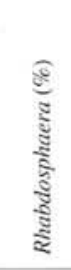 & טّ & 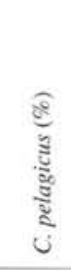 & 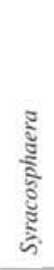 & $\frac{5}{s}$ & 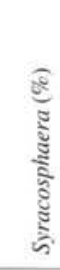 \\
\hline \multicolumn{27}{|l|}{$144-872 \mathrm{~A}-$} \\
\hline $1 \mathrm{H}-1,25-26$ & 0.25 & 152 & 28.04 & 199 & 36.72 & 1 & 0.18 & 25 & 4.61 & 115 & 21.22 & 5 & 0.92 & 3 & 0.55 & 13 & 2.40 & 1 & 0.18 & 5 & 0.92 & 0 & 0.00 & 6 & 4 & 1.11 \\
\hline $1 \mathrm{H}-1.85-86$ & 0.85 & 60 & 11.05 & 282 & 51.93 & 8 & 1.47 & 27 & 4.97 & 119 & 21.92 & 8 & 1.47 & 7 & 1.29 & 10 & 1.84 & 0 & 0.00 & 3 & 0.55 & 0 & 0.00 & 4 & 3 & 0.74 \\
\hline $1 \mathrm{H}-1,135-136$ & 1.35 & 6 & 1.13 & 337 & 63.47 & 0 & 0.00 & 33 & 6.21 & 120 & 22.60 & 3 & 0.56 & $i$ & 0.19 & 5 & 0.94 & 0 & 0.00 & 4 & 0.75 & 0 & 0.00 & 10 & 5 & 1.88 \\
\hline $1 \mathrm{H}-2,2-26$ & 1.75 & 0 & 0.00 & 232 & 43.94 & 5 & 0.95 & 121 & 22.92 & 144 & 27.27 & 2 & 0.38 & 3 & 0.57 & 7 & 1.33 & 0 & 0.00 & 2 & 0.38 & 0 & 0.00 & 0 & 3 & 0.00 \\
\hline IH-2, 8-86 & 2.35 & 0 & 0.00 & 182 & 33.39 & 0 & 0.00 & 152 & 27.89 & 157 & 28.81 & 10 & 1.83 & 3 & 0.55 & 5 & 0.92 & 0 & 0.00 & 4 & 0.73 & 0 & 0.00 & 5 & 2 & 0.92 \\
\hline $1 \mathrm{H}-2,135-136$ & 2.85 & 0 & 0.00 & 247 & 46.96 & 4 & 0.76 & 132 & 25.10 & 77 & 14.64 & 5 & 0.95 & 6 & 1.14 & 5 & 0.95 & 0 & 0.00 & i & 0.19 & 0 & 0.00 & 0 & 3 & 0.00 \\
\hline $\mathrm{IH}-3,25-26$ & 3.25 & 0 & 0.00 & 381 & 72.30 & 0 & 0.00 & 38 & 7.21 & 44 & 8.35 & 3 & 0.57 & 5 & 0.95 & 4 & 0.76 & 0 & 0.00 & 2 & 0.38 & 0 & 0.00 & 5 & 3 & 0.95 \\
\hline IH $-3,85-86$ & 3.85 & 0 & 0.00 & 348 & 67.57 & 0 & 0.00 & 72 & 13.98 & 18 & 3.50 & 6 & 1.17 & 5 & 0.97 & 4 & 0.78 & 0 & 0.00 & 2 & 0.39 & 0 & 0.00 & 2 & 2 & 0.39 \\
\hline IH-3, 135-136 & 4.35 & 0 & 0.00 & 284 & 52.79 & 0 & 0.00 & 97 & 18.03 & 10 & 1.86 & 10 & 1.86 & 11 & 2.04 & 6 & 1.12 & 0 & 0.00 & 0 & 0.00 & 0 & 0.00 & 0 & 4 & 0.00 \\
\hline $1 \mathrm{H}-4,25-26$ & 4.75 & 0 & 0.00 & 171 & 32.88 & 0 & 0.00 & 163 & 31.35 & 27 & 5.19 & 0 & 0.00 & 2 & 0.38 & 1 & 0.19 & 0 & 0.00 & i & 0.19 & 0 & 0.00 & 2 & 0 & 0.38 \\
\hline $1 \mathrm{H}-4,85-86$ & 5.35 & 0 & 0.00 & 342 & 63.93 & 0 & 0.00 & 38 & 7.10 & 0 & 0.00 & 4 & 0.75 & 5 & 0.93 & 12 & 2.24 & 0 & 0.00 & i & 0.19 & 0 & 0.00 & 2 & 0 & 0.37 \\
\hline $1 \mathrm{H}-4,135-136$ & 5.85 & 0 & 0.00 & 357 & 69.32 & 0 & 0.00 & 57 & 11.07 & 1 & 0.19 & 1 & 0.19 & 1 & 0.19 & 10 & 1.94 & 0 & 0.00 & 1 & 0.19 & 0 & 0.00 & 6 & 0 & 1.17 \\
\hline $1 \mathrm{H}-5,25-26$ & 6.25 & 0 & 0.00 & 414 & 79.31 & 2 & 0.38 & 3 & 0.57 & 0 & 0.00 & i & 0.19 & i & 0.19 & 7 & 1.34 & 1 & 0.19 & 2 & 0.38 & 0 & 0.00 & 0 & 4 & 0.00 \\
\hline $1 \mathrm{H}-5,85-86$ & 6.85 & 0 & 0.00 & 406 & 80.56 & 37 & 7.34 & 16 & 3.17 & 0 & 0.00 & 7 & 1.39 & 5 & 0.99 & 2 & 0.40 & 0 & 0.00 & 2 & 0.40 & 0 & 0.00 & 0 & 0 & 0.00 \\
\hline IH-CC & 7.30 & 0 & 0.00 & 418 & 80.23 & 40 & 7.68 & 7 & 1.34 & 0 & 0.00 & 5 & 0.96 & 11 & 2.11 & 6 & 1.15 & I & 0.19 & 4 & 0.77 & 0 & 0.00 & 4 & 0 & 0.77 \\
\hline $2 \mathrm{H}-1,0-1$ & 7.55 & 0 & 0.00 & 393 & 76.16 & 0 & 0. & 13 & 2.52 & 3 & 0.58 & 15 & 2.91 & 6 & 1.16 & 2 & 0.39 & 0 & 0.00 & 0 & 0.00 & 5 & 0.97 & 2 & 0 & 0.39 \\
\hline $2 \mathrm{H}-1,25-26$ & 7.75 & 0 & 0.00 & 412 & 80.31 & 0 & 0.00 & 8 & 1.56 & 4 & 0.78 & 8 & 1.56 & 4 & 0.78 & 2 & 0.39 & 0 & 0.00 & 0 & 0.00 & 1 & 0.19 & 3 & 1 & 0.58 \\
\hline $2 \mathrm{H}-1,85-86$ & 8.35 & 0 & 0.00 & 365 & 66.97 & 0 & 0.00 & 0 & 0.00 & 3 & 0.55 & 4 & 0.73 & 15 & 2.75 & 11 & 2.02 & 0 & 0.00 & 0 & 0.00 & 5 & 0.92 & 5 & 2 & 0.92 \\
\hline $2 \mathrm{H}-1,135-136$ & 8.85 & 0 & 0.00 & 357 & 64.32 & 0 & 0.00 & 0 & 0.00 & 0 & 0.00 & 6 & 1.08 & 12 & 2.16 & 8 & 1.44 & 0 & 0.00 & 0 & 0.00 & 5 & 0.90 & 6 & 2 & 1.08 \\
\hline
\end{tabular}


Table 4 (continued).

\begin{tabular}{|c|c|c|c|c|c|c|c|c|c|c|c|c|c|c|c|c|c|c|c|c|c|c|}
\hline $\begin{array}{l}\text { Core, section, } \\
\text { interval }(\mathrm{cm})\end{array}$ & $\frac{\sqrt[3]{5}}{\sqrt[c]{3}}$ & 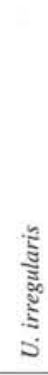 & 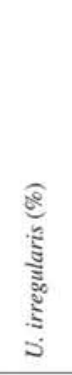 & 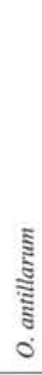 & 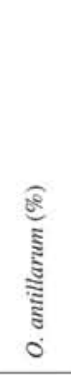 & 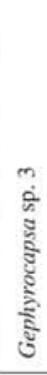 & 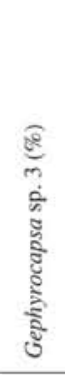 & 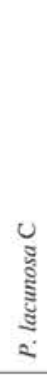 & 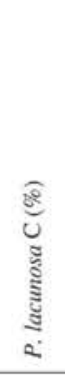 & 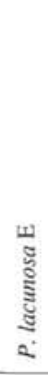 & 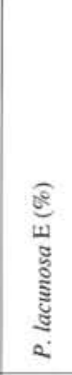 & 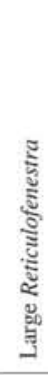 & 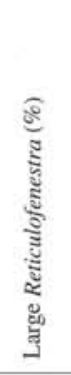 & 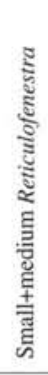 & 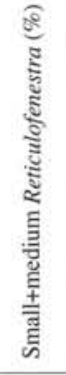 & $\begin{array}{l}\Xi \\
\vdots \\
\vdots \\
\vdots\end{array}$ & 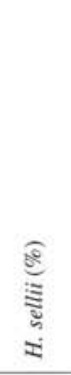 & हूँ & 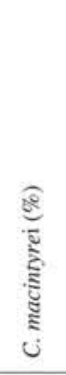 & $\frac{5}{0}$ & 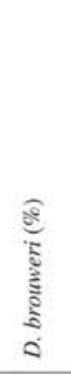 & 푱 \\
\hline \multicolumn{23}{|l|}{ 144-872A- } \\
\hline IH-1, 25-26 & 0.74 & 6 & 1.11 & $\begin{array}{l}0 \\
0\end{array}$ & 0.00 & $\begin{array}{l}7 \\
5\end{array}$ & $\begin{array}{l}1.29 \\
0.92\end{array}$ & $\begin{array}{l}0 \\
0\end{array}$ & & & & $\begin{array}{l}0 \\
0\end{array}$ & $\begin{array}{l}0.00 \\
0.00\end{array}$ & $\begin{array}{l}0 \\
0\end{array}$ & $\begin{array}{l}0.00 \\
0.00\end{array}$ & $\begin{array}{l}0 \\
0\end{array}$ & $\begin{array}{l}0.00 \\
0.00\end{array}$ & $\begin{array}{l}0 \\
0\end{array}$ & $\begin{array}{l}0.00 \\
0.00\end{array}$ & $\begin{array}{l}0 \\
0\end{array}$ & $\begin{array}{l}0.00 \\
0.00\end{array}$ & $\begin{array}{l}542 \\
543\end{array}$ \\
\hline $\begin{array}{l}\text { IH-1, 85-86 } \\
\text { IH-1,135-136 }\end{array}$ & $\begin{array}{l}0.55 \\
0.94\end{array}$ & $\begin{array}{l}7 \\
4\end{array}$ & $\begin{array}{l}1.29 \\
0.75\end{array}$ & $\begin{array}{l}0 \\
0\end{array}$ & $\begin{array}{l}0.00 \\
0.00\end{array}$ & $\begin{array}{l}5 \\
3\end{array}$ & $\begin{array}{l}0.92 \\
0.56\end{array}$ & $\begin{array}{l}0 \\
0\end{array}$ & $\begin{array}{l}0.00 \\
0.00\end{array}$ & $\begin{array}{l}0 \\
0\end{array}$ & $\begin{array}{l}0.00 \\
0.00\end{array}$ & $\begin{array}{l}0 \\
0\end{array}$ & $\begin{array}{l}0.00 \\
0.00\end{array}$ & $\begin{array}{l}0 \\
0\end{array}$ & $\begin{array}{l}0.00 \\
0.00\end{array}$ & $\begin{array}{l}0 \\
0\end{array}$ & $\begin{array}{l}0.00 \\
0.00\end{array}$ & $\begin{array}{l}0 \\
0\end{array}$ & $\begin{array}{l}0.00 \\
0.00\end{array}$ & $\begin{array}{l}0 \\
0\end{array}$ & $\begin{array}{l}0.00 \\
0.00\end{array}$ & $\begin{array}{l}543 \\
531\end{array}$ \\
\hline $1 \mathrm{H}-2,2-26$ & 0.57 & 3 & 0.57 & 2 & 0.38 & 3 & 0.57 & 1 & 0.19 & 0 & 0.00 & 0 & 0.00 & 0 & 0.00 & 0 & 0.00 & 0 & 0.00 & 0 & 0.00 & 528 \\
\hline $1 \mathrm{H}-2,8-86$ & 0.37 & 0 & 0.00 & 0 & 0.00 & 5 & 0.92 & 5 & 0.92 & 15 & 2.75 & 0 & 0.00 & 0 & 0.00 & 0 & 0.00 & 0 & 0.00 & 0 & 0.00 & 545 \\
\hline $1 \mathrm{H}-2,135-136$ & 0.57 & 4 & 0.76 & 0 & 0.00 & 4 & 0.7 & 14 & 2.66 & 24 & 4.56 & 0 & & 0 & 0.00 & 0 & 0.00 & 0 & 0.00 & 0 & & 526 \\
\hline IH $-3,25-26$ & 0.57 & 4 & 0.76 & 4 & 0.76 & 5 & 0.5 & 7 & 1.33 & 22 & 4.17 & 3 & & 0 & 0.00 & 0 & 0.00 & 0 & 0.00 & 0 & 0.00 & 527 \\
\hline IH $-3,85-86$ & 0.39 & 0 & 0.00 & 0 & 0.00 & 17 & & 18 & 3.50 & 21 & 4.08 & 0 & 0. & 0 & 0. & 0 & 0.0 & 0 & 0.00 & 0 & 0.00 & 515 \\
\hline $1 \mathrm{H}-3,135-136$ & 0.74 & 4 & 0.74 & 0 & 0.00 & 21 & 3. & 30 & 5.58 & 61 & 11.34 & 0 & 0.0 & 0 & 0.00 & 0 & 0.00 & 0 & 0.00 & 0 & 0.00 & 538 \\
\hline $1 \mathrm{H}-4,25-26$ & 0.00 & 1 & 0.19 & 0 & 0.00 & 14 & 2. & 38 & 7.3 & 87 & 16.73 & 13 & 2.5 & 0 & 0.00 & 0 & 0.00 & 0 & 0.00 & 0 & 0.00 & 520 \\
\hline $1 \mathrm{H}-4,85-86$ & 0.00 & 10 & 1. & 0 & 0.00 & 0 & 0. & 28 & 5.23 & 73 & 13.64 & 12 & 2. & 8 & 1.50 & 0 & 0.00 & 0 & 0.00 & 0 & 0.00 & 535 \\
\hline $1 \mathrm{H}-4,135-136$ & 0.00 & 5 & 0.97 & 0 & 0.00 & 0 & & 18 & 3.50 & 47 & 9.13 & 4 & 0. & 7 & 1.36 & 0 & 0.00 & 0 & 0.00 & 0 & 0.00 & 515 \\
\hline $1 \mathrm{H}-5,25-26$ & 0.77 & 0 & 0.00 & 0 & 0.00 & 0 & & 5 & 0.96 & 77 & 14.75 & 0 & 0.00 & 5 & 0.96 & 0 & 0.00 & 0 & 0.00 & 0 & 0.00 & 522 \\
\hline $1 \mathrm{H}-5,85-86$ & 0.00 & 5 & 0. & 0 & 0. & 0 & 0. & 7 & 1.39 & 17 & 3.37 & 0 & & 0 & 0.00 & 0 & 0.00 & 0 & 0.00 & 0 & 0.00 & 504 \\
\hline IH-CC & 0.8 & 0 & 0. & 0 & 0. & 0 & 0. & 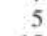 & 0. & 2 & 3 . & 0 & & 0 & 0. & 0 & 0.6 & 0 & 0 . & 0 & & 521 \\
\hline $2 \mathrm{~F}$ & 0. & 1 & & 0 & 0. & 0 & 0. & 15 & & 49 & 9. & 0 & & 0 & 0. & 2 & 0. & 9 & & 0 & 0.00 & 516 \\
\hline $2 \mathrm{H}-1,25-$ & 0.1 & 1 & 0.1 & 0 & 0. & 0 & 0. & 15 & 2. & 51 & 9.94 & 0 & 0.00 & 0 & 0.00 & 0 & 0.00 & 3 & 0.58 & 0 & 0.00 & 513 \\
\hline $2 \mathrm{H}-1,85-8$ & 0.37 & 2 & 0.37 & 0 & 0.00 & 0 & 0.0 & 32 & 5.8 & 71 & 13.03 & 0 & 0.00 & 13 & 2.39 & 4 & 0.73 & 13 & 2.39 & 0 & 0.00 & 545 \\
\hline $2 \mathrm{H}-1,135-136$ & 0.36 & 2 & 0.36 & 0 & 0.00 & 0 & 0.00 & 31 & 5.59 & 78 & 14.05 & 0 & 0.00 & 12 & 2.16 & 6 & 1.08 & 15 & 2.70 & 10 & 1.80 & 555 \\
\hline
\end{tabular}


Table 5. Quantitative distribution of Quaternary calcareous nannofossils at Site 873 (Wodejebato Guyot).

\begin{tabular}{|c|c|c|c|c|c|c|c|c|c|c|c|c|c|c|c|c|c|c|c|}
\hline $\begin{array}{l}\text { Core, section, } \\
\text { interval }(\mathrm{cm})\end{array}$ & $\begin{array}{l}\text { Depth } \\
\text { (mbsf) }\end{array}$ & 这 & 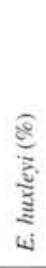 & 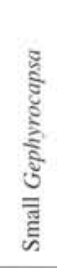 & 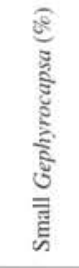 & 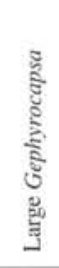 & 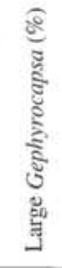 & 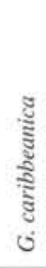 & 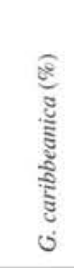 & 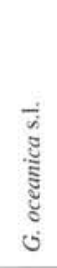 & 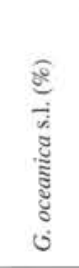 & $\begin{array}{l}\bar{z} \\
\text { š } \\
\text { i }\end{array}$ & 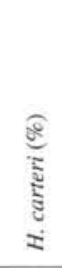 & 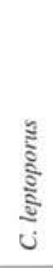 & 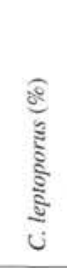 & ভ̃ & 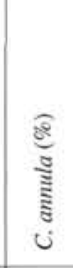 & 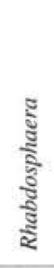 & 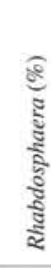 \\
\hline \multicolumn{20}{|l|}{$144-873 \mathrm{~B}-$} \\
\hline $1 \mathrm{H}-1,5-6$ & 0.05 & 0 & 0 & 323 & 61.88 & 49 & 9.39 & 28 & 5.36 & 79 & 15.13 & 5 & 0.96 & 6 & 1.15 & 6 & 1.15 & 3 & 0.57 \\
\hline $1 \mathrm{H}-1,50-51$ & 0.50 & 0 & 0 & 300 & 56.60 & 25 & 4.72 & 48 & 9.06 & 109 & 20.57 & 10 & 1.89 & 3 & 0.57 & 3 & 0.57 & 1 & 0.19 \\
\hline $1 \mathrm{H}-1,100-101$ & 1.00 & 0 & 0 & 307 & 54.43 & 31 & 5.50 & 59 & 10.46 & 85 & 15.07 & 7 & 1.24 & 6 & 1.06 & 4 & 0.71 & 0 & 0.00 \\
\hline $1 \mathrm{H}-2,5-6$ & 1.24 & 0 & 0 & 311 & 59.92 & 37 & 7.13 & 72 & 13.87 & 22 & 4.24 & 5 & 0.96 & 5 & 0.96 & 9 & 1.73 & 0 & 0.00 \\
\hline $1 \mathrm{H}-2,24-25$ & 1.48 & 0 & 0 & 252 & 48.93 & 15 & 2.91 & 85 & 16.50 & 58 & 11.26 & 12 & 2.33 & 11 & 2.14 & 6 & 1.17 & 1 & 0.19 \\
\hline $1 \mathrm{H}-2,50-51$ & 1.69 & 0 & 0 & 265 & 51.76 & 0 & 0.00 & 52 & 10.16 & 14 & 2.73 & 5 & 0.98 & 4 & 0.78 & 4 & 0.78 & 0 & 0.00 \\
\hline $1 \mathrm{H}-2,100-101$ & 2.19 & 0 & 0 & 299 & 55.78 & 5 & 0.93 & 48 & 8.96 & 13 & 2.43 & 8 & 1.49 & 5 & 0.93 & 4 & 0.75 & 4 & 0.75 \\
\hline IH $-3,5-6$ & 2.62 & 0 & 0 & 308 & 59.81 & 17 & 3.30 & 23 & 4.47 & 7 & 1.36 & 17 & 3.30 & 6 & 1.17 & 6 & 1.17 & 7 & 1.36 \\
\hline $1 \mathrm{H}-3,50-51$ & 3.07 & 0 & 0 & 190 & 37.18 & 3 & 0.59 & 22 & 4.31 & 15 & 2.94 & 9 & 1.76 & 8 & 1.57 & 5 & 0.98 & 4 & 0.78 \\
\hline $1 \mathrm{H}-3,100-101$ & 3.57 & 0 & 0 & 221 & 42.50 & 0 & 0.00 & 23 & 4.42 & 22 & 4.23 & 21 & 4.04 & 13 & 2.50 & 6 & 1.15 & 0 & 0.00 \\
\hline IH- $4,5-6$ & 4.02 & 0 & 0 & 227 & 43.82 & 0 & 0.00 & 10 & 1.93 & 7 & 1.35 & 12 & 2.32 & 4 & 0.77 & 0 & 0.00 & 2 & 0.39 \\
\hline $1 \mathrm{H}-4,50-51$ & 4.47 & 0 & 0 & 207 & 38.33 & 0 & 0.00 & I & 0.19 & 0 & 0,00 & 12 & 2.22 & 7 & 1.30 & 55 & 10.19 & 0 & 0.00 \\
\hline $1 \mathrm{H}-4,100-101$ & 4.97 & 0 & 0 & 210 & 38.89 & 0 & 0.00 & 0 & 0.00 & 0 & 0.00 & 10 & 1.85 & 6 & 1.11 & 45 & 8.33 & 2 & 0.37 \\
\hline
\end{tabular}

and discussion. Isabella Raffi and Wuchang Wei are acknowledged for the constructive critical review of the manuscript. Research was supported by CNR funds.

\section{REFERENCES}

Berggren, W.A., Kent, D.V., Flynn, J.J., and Van Couvering, J.A., 1985. Cenozoic geochronology. Geol. Soc. Am. Bull., 96:1407-1418.

Bukry, D., 1975. Coccolith and silicoflagellate stratigraphy, northwestern Pacific Ocean, Deep Sea Drilling Project Leg 32. In Larson, R.L., Moberly, R., et al., Init. Repts. DSDP, 32: Washington (U.S. Govt. Printing Office), 677-701.

Castradori, D., 1993a. Calcareous nannofossil biostratigraphy and biochronology in eastern Mediterranean deep-sea cores. Riv. Ital. Paleontol. Stratigr. 99:107-126.

, 1993b. Calcareous nannofossils and the origin of eastern Mediterranean sapropels. Paleoceanography, 8:459-471.

Gartner, S., 1977. Calcareous nannofossil biostratigraphy and revised zonation of the Pleistocene. Mar. Micropaleontol., 2:1-25.

1988. Paleoceanography of the mid-Pleistocene. Mar. Micropaleontol., 13:23-46.

Hills, S.J., and Thierstein, H.R., 1989. Plio-Pleistocene calcareous plankton biochronology. Mar. Micropaleontol., 14:67-96.

Matsuoka, H., and Okada, H., 1989. Quantitative analysis of Quaternary nannoplankton in the subtropical northwestern Pacific Ocean. Mar: Micropaleontol., 14:97-118.

. 1990. Time-progressive morphometric changes of the genus Gephyrocapsa in the Quaternary sequence of the tropical Indian Ocean, Site 709. In Duncan, R.A., Backman, J., Peterson, L.C., et al., Proc. ODP, Sci. Results, 115: College Station, TX (Ocean Drilling Program), 255-270.

Premoli Silva, I., Haggerty, J., Rack, F., et al., 1993. Proc. ODP, Init. Repts. 144: College Station, TX (Ocean Drilling Program).

Pujos, A., 1985a. Nannofossils from Quaternary deposits in the high-productivity area of the central equatorial Pacific, Deep Sea Drilling Project Leg 85. In Mayer, L., Theyer, F., Thomas, E., et al., Init. Repts. DSDP, 85: Washington (U.S. Govt. Printing Office), 553-579.

, 1985b. Quaternary nannofossils from Goban Spur, eastern North Atlantic Ocean, Deep Sea Drilling Project Holes 548 and 549A. In de Graciansky, P.C., Poag, C.W., et al., Init. Repts. DSDP, 80: Washington (U.S. Govt. Printing Office), 767-792.
Raffi, I., Backman, J., Rio, D., and Shackleton, N.J., 1993. Plio-Pleistocene nannofossil biostratigraphy and calibration to oxygen isotopes stratigraphies from Deep Sea Drilling Project Site 607 and Ocean Drilling Program Site 677. Paleoceanography, 8:387-408.

Rio, D., 1982. The fossil distribution of coccolithophore genus Gephyrocapsa Kamptner and related Plio-Pleistocene chronostratigraphic problems. In Prell, W.L., Gardner, J.V., et al., Init. Repts. DSDP, 68: Washington (U.S. Govt. Printing Office), 325-343.

Rio, D., Raffi, I., and Villa, G., 1990. Pliocene-Pleistocene calcareous nannofossil distribution patterns in the Western Mediterranean. In Kastens, K.A., Mascle, J., et al., Proc. ODP, Sci. Results, 107: College Station, TX (Ocean Drilling Program), 513-533.

Sato, T., and Takayama, T., 1990. A stratigraphically significant new species of the calcareous nannofossil Reticulofenestra asanoi. In Ishizaki, K., and Saito, T. (Eds.), Centenary of Japanese Micropaleontology: Tokyo (Terra Sci. Publ.), 457-460.

Shackleton, N.J., Berger, A., and Peltier, W.R., 1990. An alternative astronomical calibration of the lower Pleistocene time scale based on ODP Site 677. Trans. R. Soc. Edinburgh: Earth Sci., 81:251-261.

Takayama, T., and Sato, T., 1987. Coccolith biostratigraphy of the North Atlantic Ocean, Deep Sea Drilling Project Leg 94. In Ruddiman, W.F., Kidd, R.B., Thomas, E., et al., Init. Repts. DSDP, 94 (Pt. 2): Washington (U.S. Govt. Printing Office), 651-702.

Thierstein, H.R., Geitzenauer, K., Molfino, B., and Shackleton, N.J., 1977. Global synchroneity of late Quaternary coccolith datum levels: validation by oxygen isotopes. Geology, 5:400-404.

Wei, W., 1993. Calibration of Upper Pliocene-Lower Pleistocene nannofossil events with oxygen isotope stratigraphy. Paleoceanography, 8:85-99.

\footnotetext{
'Abbreviations for names of organizations and publications in ODP reference lists follow the style given in Chemical Abstracts Service Source Index (published by American Chemical Society).
}

Date of initial receipt: 1 February 1994

Date of acceptance: 20 June 1994

Ms 144SR-004 
Table 5 (continued).

\begin{tabular}{|c|c|c|c|c|c|c|c|c|c|c|c|c|c|c|c|c|c|c|c|c|c|c|c|c|}
\hline 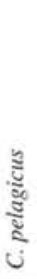 & 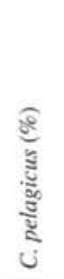 & 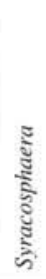 & 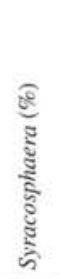 & $\frac{3}{3}$ & $\frac{\sqrt[3]{3}}{\sqrt[3]{3}}$ & 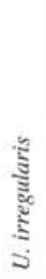 & 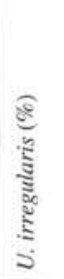 & 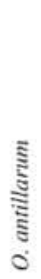 & 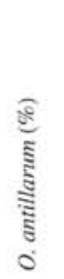 & 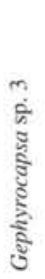 & 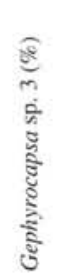 & 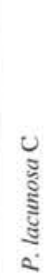 & 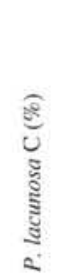 & 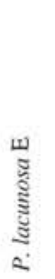 & 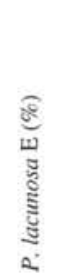 & 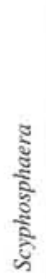 & 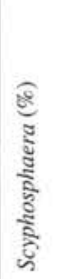 & 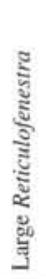 & 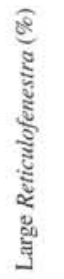 & $\begin{array}{l}\vdots \\
\vdots \\
2\end{array}$ & 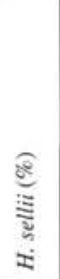 & 产 & 怤 & 豆 \\
\hline 0 & 0.00 & 5 & 0.96 & 2 & 0.38 & 3 & 0.57 & 0 & 0.00 & 0 & 0.00 & 3 & 0.57 & 9 & 1.72 & 0 & 0.00 & 0 & 0.00 & I & 0.19 & 0 & 0.00 & 522 \\
\hline 0 & 0.00 & 6 & 1.13 & 2 & 0.38 & 4 & 0.75 & 0 & 0.00 & 0 & 0.00 & 5 & 0.94 & 8 & 1.51 & 0 & 0.00 & 0 & 0.00 & 6 & 1.13 & 0 & 0.00 & 530 \\
\hline 0 & 0.00 & 5 & 0.89 & 1 & 0.18 & 5 & 0.89 & 1 & 0.18 & 0 & 0.00 & 13 & 2.30 & 35 & 6.21 & 0 & 0.00 & 0 & 0.00 & 5 & 0.89 & 0 & 0.00 & 564 \\
\hline 0 & 0.00 & 2 & 0.39 & 1 & 0.19 & 0 & 0.00 & 2 & 0.39 & 0 & 0.00 & 19 & 3.66 & 31 & 5.97 & 0 & 0.00 & 0 & 0.00 & 3 & 0.58 & 0 & 0.00 & 519 \\
\hline 0 & 0.00 & 3 & 0.58 & 2 & 0.39 & 0 & 0.00 & 0 & 0.00 & 0 & 0.00 & 28 & 5.44 & 39 & 7.57 & 0 & 0.00 & 0 & 0.00 & 3 & 0.58 & 0 & 0.00 & 515 \\
\hline 0 & 0.00 & 0 & 0.00 & 2 & 0.39 & 1 & 0.20 & 0 & 0.00 & 0 & 0.00 & 44 & 8.59 & 116 & 22.66 & 0 & 0.00 & 3 & 0.59 & 2 & 0.39 & 0 & 0.00 & 512 \\
\hline 0 & 0.00 & 5 & 0.93 & 1 & 0.19 & $i$ & 0.19 & 0 & 0.00 & 0 & 0.00 & 37 & 6.90 & 101 & 18.84 & 0 & 0.00 & 2 & 0.37 & 3 & 0.56 & 0 & 0.00 & 536 \\
\hline I & 0.19 & 10 & 1.94 & 1 & 0.19 & 1 & 0.19 & 0 & 0.00 & 0 & 0.00 & 27 & 5.24 & 82 & 15.92 & 0 & 0.00 & 0 & 0.00 & 2 & 0.39 & 0 & 0.00 & 515 \\
\hline 0 & 0.00 & 0 & 0.00 & 3 & 0.59 & 0 & 0,00 & 0 & 0.00 & 0 & 0.00 & 57 & 11.15 & 193 & 37.77 & 0 & 0.00 & 0 & 0.00 & 2 & 0.39 & 0 & 0.00 & 511 \\
\hline 0 & 0.00 & 3 & 0.58 & 0 & 0.00 & 2 & 0.38 & 2 & 0.38 & 0 & 0.00 & 89 & 17.12 & 102 & 19.62 & 2 & 0.38 & 0 & 0.00 & 3 & 0.58 & 11 & 2.12 & 520 \\
\hline 7 & 1.35 & 3 & 0.58 & 2 & 0.39 & 0 & 0.00 & 0 & 0.00 & 0 & 0.00 & 52 & 10.04 & 175 & 33.78 & 0 & 0.00 & 0 & 0.00 & 2 & 0.39 & 15 & 2.90 & 518 \\
\hline 2 & 0.37 & 6 & 1.11 & 2 & 0.37 & 0 & 0.00 & 3 & 0.56 & 0 & 0.00 & 103 & 19.07 & 114 & 21.11 & 1 & 0.19 & 0 & 0.00 & 11 & 2.04 & 15 & 2.78 & 540 \\
\hline 8 & 1.48 & 5 & 0.93 & 2 & 0.37 & 2 & 0.37 & 4 & 0.74 & 0 & 0.00 & 105 & 19.44 & 120 & 22.22 & 1 & 0.19 & 0 & 0.00 & 12 & 2.22 & 17 & 3.15 & 540 \\
\hline
\end{tabular}

Site 880

Takuyo-Daisan
Site 871

Limalok
Site 872

Lo-En
Site 873

Wodejebato

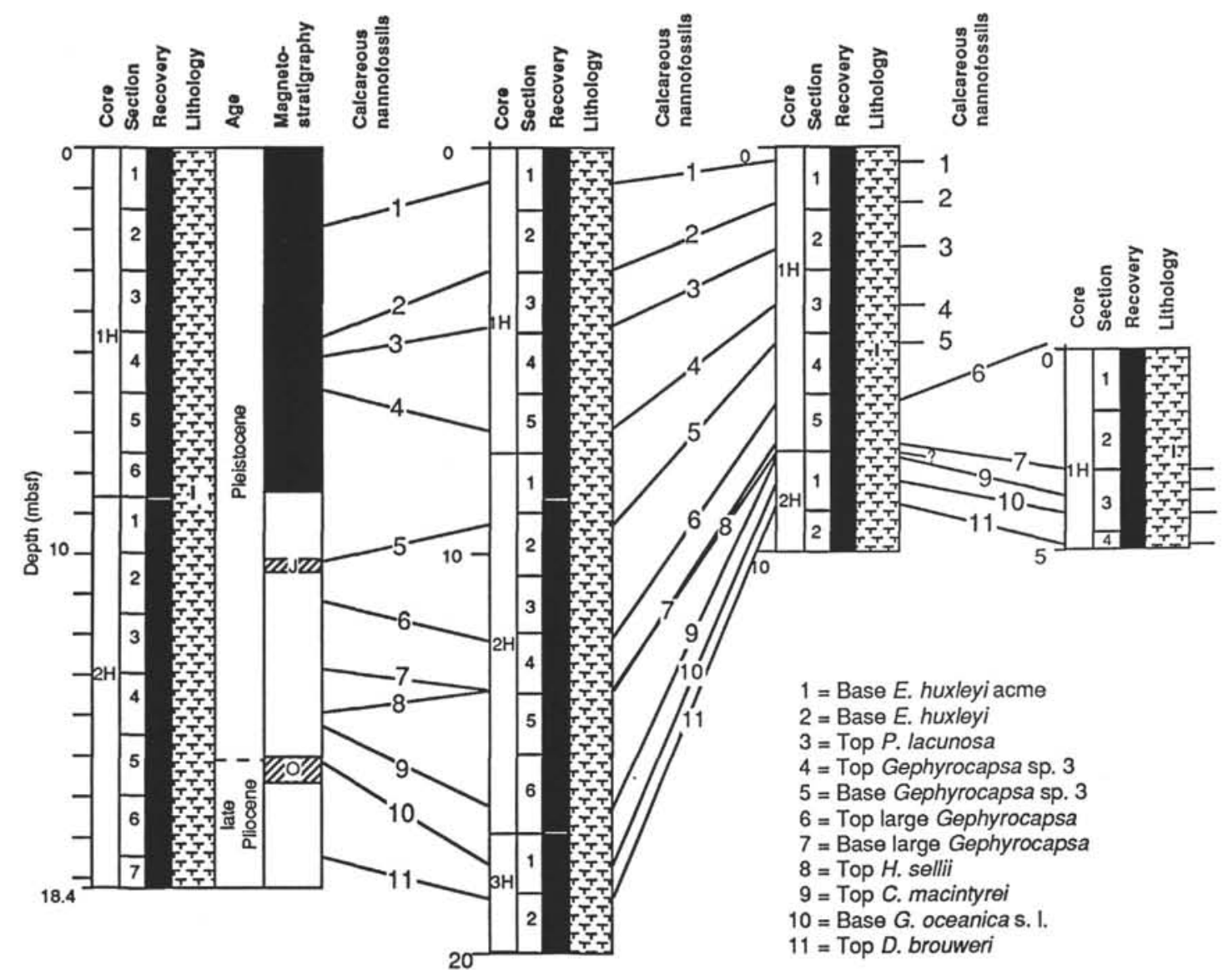

Figure 6. Correlation of Sites $800,871,872$, and 873 based on nannofossil quantitative biostratigraphy. 
Table 6. Ages (in k.y.) of Quaternary nannofossil events at Sites $880,871,872$, and 873 , as published by previous workers.

\begin{tabular}{|c|c|c|c|c|c|c|c|c|c|c|c|c|c|c|}
\hline Author & Area & 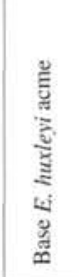 & 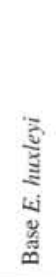 & 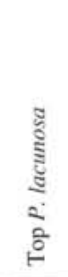 & 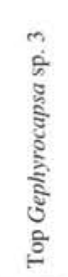 & 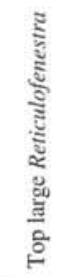 & 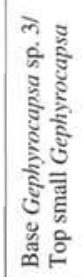 & 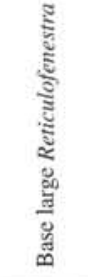 & 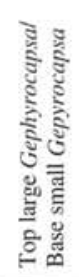 & 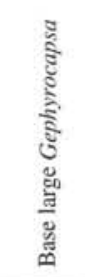 & $\begin{array}{l}\vdots \\
\vdots \\
\vdots \\
\vdots \\
\vdots \\
\vdots\end{array}$ & 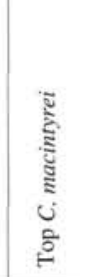 & 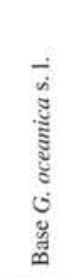 & 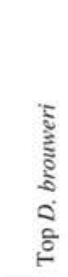 \\
\hline $\begin{array}{l}\text { Bukry, } 1975 \\
\text { Gartner, } 1977 \\
\text { Thierstein et al., } 1977 \\
\text { Berggren et al., } 1985 \\
\text { Hills and Thierstein, } 1989 \\
\text { Rio et al., 1990 } \\
\text { Matsuoka and Okada, } 1989 \\
\text { Takayama and Sato, } 1987 \\
\text { Castradori, 1993a } \\
\text { Pujos, 1985b } \\
\text { Pujos, 1985a } \\
\text { Raffi et al., 1993 } \\
\text { Raffi et al.. 1993 } \\
\text { Wei, 1993* }\end{array}$ & $\begin{array}{l}\text { Northwest Pacific } \\
\text { Caribbean and Pacific } \\
\text { Global } \\
\text { Global } \\
\text { Global } \\
\text { West Mediterranean } \\
\text { Pacific } \\
\text { North Atlantic } \\
\text { East Mediterranean } \\
\text { North Atantic } \\
\text { Central equatorial Pacific } \\
\text { Pacific } \\
\text { North Atlantic } \\
\text { Global }\end{array}$ & $\begin{array}{c}70 \\
73-85\end{array}$ & $\begin{array}{l}200 \\
270 \\
268 \\
275 \\
230 \\
\\
270 \\
240 \\
265\end{array}$ & $\begin{array}{l}300 \\
440 \\
458 \\
474 \\
350 \\
460 \\
390 \\
468 \\
475\end{array}$ & 584 & $\begin{array}{l}810 \\
830\end{array}$ & $\begin{array}{r}920 \\
900 \\
950 \\
920 \\
890 \\
944 \\
\\
1028 \\
957\end{array}$ & $\begin{array}{l}1020- \\
1170\end{array}$ & $\begin{array}{l}1240 \\
1227 \\
1230\end{array}$ & $\begin{array}{c}1470 \\
1479 \\
1440- \\
1530\end{array}$ & $\begin{array}{c}1200 \\
1190 \\
1200 \\
1230 \\
1470 \\
1241 \\
1260- \\
1440\end{array}$ & $\begin{array}{l}1570 \\
1650 \\
1500 \\
1597 \\
1640 \\
1600- \\
1640\end{array}$ & $\begin{array}{l}1670 \\
1700 \\
1680\end{array}$ & $\begin{array}{r}2000 \\
1950 \\
1950- \\
1960\end{array}$ \\
\hline
\end{tabular}

"Ages after Shackleton et al. (1990).

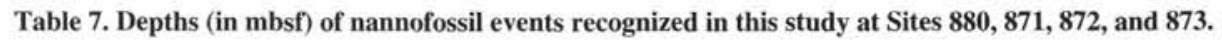

\begin{tabular}{|c|c|c|c|c|c|c|c|c|c|c|c|c|c|c|}
\hline Site & Guyot & 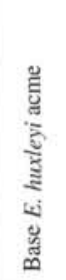 & 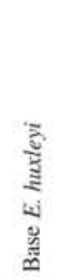 & 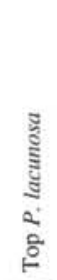 & 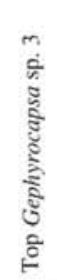 & 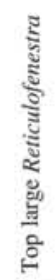 & 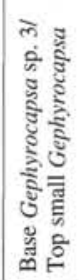 & 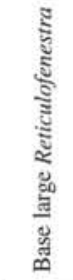 & 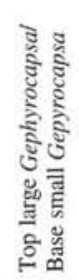 & 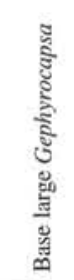 & 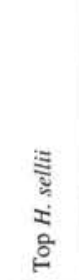 & 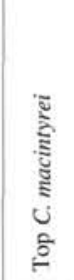 & 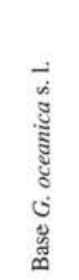 & 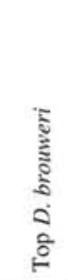 \\
\hline 871 & Limalok & 0.82 & 2.9 & 5. & 6.8 & 8.85 & 9.25 & 11. & 12. & 13.35 & 13.35 & 16.35 & 17.25 & 18.35 \\
\hline 872 & Lo-En & 0.25 & 1.35 & 2.35 & 3.85 & 3.2 & 4.75 & 8.85 & 6.2 & 7.3 & 7.55 & 7.55 & 8.35 & 8. \\
\hline 873 & Wodejebato & & & & & & & & & 3.07 & & 3.57 & 4.02 & 4.97 \\
\hline 880 & Takuyo-Daisan & 1.8 & 4.8 & 5.2 & 5.8 & 6.39 & 10.2 & 12.17 & 11.59 & 13.17 & 14.37 & 14.57 & 15.97 & 16.37 \\
\hline
\end{tabular}

